\title{
On Brane Solutions Related to Non-Singular Kac-Moody Algebras
}

Vladimir D. IVASHCHUK and Vitaly N. MELNIKOV

Center for Gravitation and Fundamental Metrology, VNIIMS, 46 Ozyornaya Str., Moscow 119361, Russia

Institute of Gravitation and Cosmology, Peoples' Friendship University of Russia, 6 Miklukho-Maklaya Str., Moscow 117198, Russia

E-mail: ivashchuk@mail.ru,melnikov@phys.msu.ru

Received October 01, 2008, in final form June 15, 2009; Published online July 07, 2009

doi:10.3842/SIGMA.2009.070

\begin{abstract}
A multidimensional gravitational model containing scalar fields and antisymmetric forms is considered. The manifold is chosen in the form $M=M_{0} \times M_{1} \times \cdots \times M_{n}$, where $M_{i}$ are Einstein spaces $(i \geq 1)$. The sigma-model approach and exact solutions with intersecting composite branes (e.g. solutions with harmonic functions, $S$-brane and black brane ones) with intersection rules related to non-singular Kac-Moody (KM) algebras (e.g. hyperbolic ones) are reviewed. Some examples of solutions, e.g. corresponding to hyperbolic KM algebras: $H_{2}(q, q), A E_{3}, H A_{2}^{(1)}, E_{10}$ and Lorentzian KM algebra $P_{10}$ are presented.
\end{abstract}

Key words: Kac-Moody algebras; $S$-branes; black branes; sigma-model; Toda chains

2000 Mathematics Subject Classification: 17B67; 17B81; 83E15; 83E50; 83F05; $81 \mathrm{~T} 30$

\section{Introduction}

Kac-Moody (KM) Lie algebras [1, 2, 3] play a rather important role in different areas of mathematical physics (see $[3,4,5,6]$ and references therein).

We recall that KM Lie algebra is a Lie algebra generated by the relations [3]

$$
\begin{aligned}
& {\left[h_{i}, h_{j}\right]=0, \quad\left[e_{i}, f_{j}\right]=\delta_{i j} h_{j},} \\
& {\left[h_{i}, e_{j}\right]=A_{i j} e_{j}, \quad\left[h_{i}, f_{j}\right]=-A_{i j} f_{j},} \\
& \left(\operatorname{ad} e_{i}\right)^{1-A_{i j}}\left(e_{j}\right)=0 \quad(i \neq j), \\
& \left(\operatorname{ad} f_{i}\right)^{1-A_{i j}}\left(f_{j}\right)=0 \quad(i \neq j) .
\end{aligned}
$$

Here $A=\left(A_{i j}\right)$ is a generalized Cartan matrix, $i, j=1, \ldots, r$, and $r$ is the rank of the KM algebra. It means that all $A_{i i}=2 ; A_{i j}$ for $i \neq j$ are non-positive integers and $A_{i j}=0$ implies $A_{j i}=0$.

In what follows the matrix $A$ is restricted to be non-degenerate (i.e. $\operatorname{det} A \neq 0$ ) and symmetrizable i.e. $A=B \mathcal{D}$, where $B$ is a symmetric matrix and $\mathcal{D}$ is an invertible diagonal matrix $\left(\mathcal{D}\right.$ may be chosen in such way that all its entries $\mathcal{D}_{i i}$ are positive rational numbers [3]). Here we do not consider singular KM algebras with $\operatorname{det} A=0$, e.g. affine ones. Recall that affine KM algebras are of much interest for conformal field theories, superstring theories etc. $[4,7]$.

In the case when $A$ is positive definite (the Euclidean case) we get ordinary finite dimensional Lie algebras [3, 4]. For non-Euclidean signatures of $A$ all KM algebras are infinite-dimensional. Among these the Lorentzian KM algebras with pseudo-Euclidean signatures $(-,+, \ldots,+)$ for the Cartan matrix $A$ are of current interest, since they contain a subclass of the so-called hyperbolic KM algebras widely used in modern mathematical physics. Hyperbolic KM algebras 
are by definition Lorentzian Kac-Moody algebras with the property that removing any node from their Dynkin diagram leaves one with a Dynkin diagram of the affine or finite type. The hyperbolic KM algebras can be completely classified [8,9] and have rank $2 \leq r \leq 10$. For $r \geq 3$ there is a finite number of hyperbolic algebras. For rank 10, there are four algebras, known as $E_{10}, B E_{10}, C E_{10}$ and $D E_{10}$. Hyperbolic $\mathrm{KM}$ algebras appeared in ordinary gravity [10] $\left(\mathcal{F}_{3}=A E_{3}=H_{3}\right)$, supergravity: [11, 12] $\left(E_{10}\right),[13]\left(\mathcal{F}_{3}\right)$, strings [14] etc.

The growth of interest in hyperbolic algebras in theoretical and mathematical physics appeared in 2001 after the publication of Damour and Henneaux [15] devoted to a description of chaotic (BKL-type [16]) behaviour near the singularity in string inspired low energy models (e.g. supergravitational ones) [17] (see also [18]). It should be noted that these results were based on a billiard approach in multidimensional cosmology with different matter sources (for $D=4$ suggested by Chitre [19]) elaborated in our papers [20, 21, 22, 23, 24] (for a review see also $[25,26])$.

The description of oscillating behaviour near the singularity in $D=11$ supergravity [27] (which is related to $M$-theory [28, 29]) in terms of motion of a point-like particle in a 9dimensional billiard (of finite volume) corresponding to the Weyl chamber of the hyperbolic KM algebra $E_{10}$ inspired another description of $D=11$ supergravity in [30]: a formal "small tension" expansion of $D=11$ supergravity near a space-like singularity was shown to be equivalent (at least up to 30th order in height) to a null geodesic motion in the infinite dimensional coset space $\mathcal{E}_{10} / K\left(\mathcal{E}_{10}\right)$ (here $K\left(\mathcal{E}_{10}\right)$ is the maximal compact subgroup of the hyperbolic KacMoody group $\left.\mathcal{E}_{10}(R)\right)$.

Recall that $E_{10} \mathrm{KM}$ algebra is an over-extension of the finite dimensional Lie algebra $E_{8}$, i.e. $E_{10}=E_{8}^{++}$. But there is another extension of $E_{8}$ - the so-called the very extended KacMoody algebra of the $E_{8}$ algebra - called $E_{11}=E_{8}^{+++}$. (To get an understanding of very extended algebras and some of their properties see [31] and references therein). It has been proposed by $\mathrm{P}$. West that the Lorentzian (non-hyperbolic) KM algebra $E_{11}$ is responsible for a hidden algebraic structure characterizing 11-dimensional supergravity [32]. The same very extended algebra occurs in $I I A$ [32] and $I I B$ supergravities [33]. Moreover, it was conjectured that an analogous hidden structure is realized in the effective action of the bosonic string (with the KM algebra $k_{27}=D_{24}^{+++}$) [32] and also for pure $D$ dimensional gravity (with the KM algebra $\left.A_{D-3}^{+++}[34]\right)$. It has been suggested in [35] that all the so-called maximally oxidised theories (see also [6]), possess the very extension $G^{+++}$of the simple Lie algebra $G$. It was shown in [36] that the BPS solutions of the oxidised theory of a simply laced group $\mathcal{G}$ form representations of a subgroup of the Weyl transformations of the algebra $G^{+++}$.

In this paper we briefly review another possibility for utilizing non-singular (e.g. hyperbolic) KM algebras suggested in three of our papers [37, 38, 39]. This possibility (implicitly assumed also in $[40,41,42,43,44,45,46])$ is related to certain classes of exact solutions describing intersecting composite branes in a multidimensional gravitational model containing scalar fields and antisymmetric forms defined on (warped) product manifolds $M=M_{0} \times M_{1} \times \cdots \times M_{n}$, where Einstein factor spaces $M_{i}(i \geq 1)$ are Ricci-flat (at least) for $i \geq 2$. From a pure mathematical point of view these solutions may be obtained from sigma-models or Toda chains corresponding to certain non-singular KM algebras. The information about the (hidden) KM algebra is encoded in intersection rules which relate the dimensions of brane intersections with non-diagonal components of the generalized Cartan matrix $A$ [47]. We deal here with generalized Cartan matrices of the form

$$
A_{s s^{\prime}} \equiv \frac{2\left(U^{s}, U^{s^{\prime}}\right)}{\left(U^{s^{\prime}}, U^{s^{\prime}}\right)}, \quad s, s^{\prime} \in S
$$

with $\left(U^{s}, U^{s}\right) \neq 0$, for all $s \in S$ (S is a finite set). Here $U^{s}$ are the so-called brane (co-)vectors. They are linear functions on $\mathbb{R}^{N}$, where $N=n+l$ and $l$ is the number of scalar fields. The 
indefinite scalar product $(\cdot, \cdot)[48]$ is defined on $\left(\mathbb{R}^{N}\right)^{*}$ and has the signature $(-1,+1, \ldots,+1)$ when all scalar fields have positive kinetic terms, i.e. there are no phantoms (or ghosts). The matrix $A$ is symmetrizable. $U^{s}$-vectors may be put in one-to-one correspondence with simple roots $\alpha_{s}$ of the generalized KM algebra after a suitable normalizing. For indecomposable $A$ (when the KM algebra is simple) the matrices $\left(\left(U^{s}, U^{s^{\prime}}\right)\right)$ and $\left(\left(\alpha_{s} \mid \alpha_{s^{\prime}}\right)\right)$ are proportional to each other. Here $(\cdot \mid \cdot)$ is a non-degenerate bilinear symmetric form on a root space obeying $\left(\alpha_{s} \mid \alpha_{s}\right)>0$ for all simple roots $\alpha_{s}[3]$.

We note that the minisuperspace bilinear form $(\cdot, \cdot)$ coming from multidimensional gravitational model [48] "does not know" about the definition of $(\cdot \mid \cdot)$ in [3] and hence there exist physical examples where all $\left(U^{s}, U^{s}\right)$ are negative. Some examples of this are given below in Section 5. For $D=11$ supergravity and ten dimensional $I I A, I I B$ supergravities all $\left(U^{s}, U^{s}\right)=2$ $[47,49]$ and corresponding KM algebras are simply laced. It was shown in our papers $[22,23,24]$ that the inequality $\left(U^{s}, U^{s}\right)>0$ is a necessary condition for the formation of the billiard wall (in one approaches the singularity) by the $s$-th matter source (e.g. a fluid component or a brane).

The scalar products for brane vectors $U^{s}$ were found in [48] (for the electric case see also $[50,51,52])$

$$
\left(U^{s}, U^{s^{\prime}}\right)=d_{s s^{\prime}}+\frac{d_{s} d_{s^{\prime}}}{2-D}+\chi_{s} \chi_{s^{\prime}}\left\langle\lambda_{s}, \lambda_{s^{\prime}}\right\rangle
$$

where $d_{s}$ and $d_{s^{\prime}}$ are dimensions of the brane worldvolumes corresponding to branes $s$ and $s^{\prime}$ respectively, $d_{s s^{\prime}}$ is the dimension of intersection of the brane worldvolumes, $D$ is the total space-time dimension, $\chi_{s}=+1,-1$ for electric or magnetic brane respectively, and $\left\langle\lambda_{s}, \lambda_{s^{\prime}}\right\rangle$ is the non-degenerate scalar product of the $l$-dimensional dilatonic coupling vectors $\lambda_{s}$ and $\lambda_{s^{\prime}}$ corresponding to branes $s$ and $s^{\prime}$.

Relations (1.1), (1.2) define the brane intersection rules [47]

$$
d_{s s^{\prime}}=d_{s s^{\prime}}^{o}+\frac{1}{2} K_{s^{\prime}} A_{s s^{\prime}},
$$

$s \neq s^{\prime}$, where $K_{s}=\left(U^{s}, U^{s}\right)$ and

$$
d_{s s^{\prime}}^{o}=\frac{d_{s} d_{s^{\prime}}}{D-2}-\chi_{s} \chi_{s^{\prime}}\left\langle\lambda_{s}, \lambda_{s^{\prime}}\right\rangle
$$

is the dimension of the so-called orthogonal (or $\left(A_{1} \oplus A_{1}\right)$-) intersection of branes following from the orthogonality condition [48]

$$
\left(U^{s}, U^{s^{\prime}}\right)=0,
$$

$s \neq s^{\prime}$. The orthogonality relations (1.4) for brane intersections in the non-composite electric case were suggested in $[50,51]$ and for the composite electric case in [52].

Relations (1.2) and (1.3) were derived in [48] for rather general assumptions: the branes were composite, the number of scalar fields $l$ was arbitrary as well as the signature of the bilinear form $\langle\cdot, \cdot\rangle$ (or, equivalently, the signature of the kinetic term for scalar fields), (Einstein) factor spaces $M_{i}$ had arbitrary dimensions $d_{i}$ and signatures. The intersection rules (1.3) appeared earlier (in different notations) in $[53,54,55]$ when all $d_{i}=1(i>0)$ and $\langle\cdot, \cdot\rangle$ was positive definite (in $[53,54] l=1$ and total space-time had a pseudo-Euclidean signature). The intersection rules $(1.3)$ were also used in $[56,57,58,47]$ in the context of intersecting black brane solutions.

It was proved in [59] that the target space of the sigma model describing composite electromagnetic brane configurations on the product of several Ricci-flat spaces is a homogeneous (coset) space $G / H$. It is locally symmetric (i.e. the Riemann tensor is covariantly constant: $\nabla$ Riem $=0)$ if and only if

$$
\left(U^{s}-U^{s^{\prime}}\right)\left(U^{s}, U^{s^{\prime}}\right)=0
$$


for all $s$ and $s^{\prime}$, i.e. when any two brane vectors $U^{s}$ and $U^{s^{\prime}}, s \neq s^{\prime}$, are either coinciding $U^{s}=U^{s^{\prime}}$ or orthogonal $\left(U^{s}, U^{s^{\prime}}\right)=0$ (for two electric branes and $l=1$ see also [60]).

Now relations for brane vectors $U^{s}(1.1)$ and (1.2) (with $U^{s}$ being identified with roots $\alpha_{s}$ ) are widely used in the $G^{+++}$-approach $[36,6]$. The orthogonality condition (1.4) describing the intersection of branes [50, 51, 52, 48] was rediscovered in [49] (for some particular intersecting configurations of M-theory it was done in [61]). It was found in the context of $G^{+++}$-algebras that the intersection rules for extremal branes are encoded in orthogonality conditions between the various roots from which the branes arise, i.e. $\left(\alpha_{s} \mid \alpha_{s^{\prime}}\right)=0, s \neq s^{\prime}$, where $\alpha_{s}$ should be real positive roots ("real" means that $\left(\alpha_{s} \mid \alpha_{s}\right)>0$ ). In [49] another condition on brane, root vectors was found: $\alpha_{s}+\alpha_{s^{\prime}}$ should not be a root, $s \neq s^{\prime}$. The last condition is trivial for M-theory and for $I I A$ and $I I B$ supergravities, but for low energy effective actions of heterotic strings it forbids certain intersections that does not take place due to non-zero contributions of Chern-Simons terms.

It should be noted that the orthogonality relations for brane intersections (1.4) which appeared in 1996-97, were not well understood by the superstring community at that time. The standard intersection rules (1.3) gave back the well-known zero binding energy configurations preserving some supersymmetries. These brane configurations were originally derived from supersymmetry and duality arguments (see for example [62, 63, 64] and reference therein) or by using a no-force condition (suggested for M-branes in [65]).

\section{The model}

\subsection{The action}

We consider the model governed by action

$$
\begin{aligned}
S= & \frac{1}{2 \kappa^{2}} \int_{M} d^{D} z \sqrt{|g|}\left\{R[g]-2 \Lambda-h_{\alpha \beta} g^{M N} \partial_{M} \varphi^{\alpha} \partial_{N} \varphi^{\beta}\right. \\
& \left.-\sum_{a \in \Delta} \frac{\theta_{a}}{n_{a} !} \exp \left[2 \lambda_{a}(\varphi)\right]\left(F^{a}\right)_{g}^{2}\right\}+S_{G H},
\end{aligned}
$$

where $g=g_{M N} d z^{M} \otimes d z^{N}$ is the metric on the manifold $M, \operatorname{dim} M=D, \varphi=\left(\varphi^{\alpha}\right) \in \mathbb{R}^{l}$ is a vector of dilatonic scalar fields, $\left(h_{\alpha \beta}\right)$ is a non-degenerate symmetric $l \times l$ matrix $(l \in \mathbb{N})$, $\theta_{a} \neq 0$

$$
F^{a}=d A^{a}=\frac{1}{n_{a} !} F_{M_{1} \ldots M_{n_{a}}}^{a} d z^{M_{1}} \wedge \cdots \wedge d z^{M_{n_{a}}}
$$

is an $n_{a}$-form $\left(n_{a} \geq 2\right)$ on a $D$-dimensional manifold $M, \Lambda$ is a cosmological constant and $\lambda_{a}$ is a 1 -form on $\mathbb{R}^{l}: \lambda_{a}(\varphi)=\lambda_{a \alpha} \varphi^{\alpha}, a \in \boldsymbol{\Delta}, \alpha=1, \ldots, l$. In (2.1) we denote $|g|=\left|\operatorname{det}\left(g_{M N}\right)\right|$,

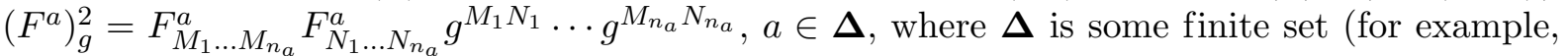
of positive integers), and $S_{\mathrm{GH}}$ is the standard Gibbons-Hawking boundary term [66]. In models with one time all $\theta_{a}=1$ when the signature of the metric is $(-1,+1, \ldots,+1) . \quad \kappa^{2}$ is the multidimensional gravitational constant.

\subsection{Ansatz for composite branes}

Let us consider the manifold

$$
M=M_{0} \times M_{1} \times \cdots \times M_{n},
$$


with the metric

$$
g=e^{2 \gamma(x)} \hat{g}^{0}+\sum_{i=1}^{n} e^{2 \phi^{i}(x)} \hat{g}^{i},
$$

where $g^{0}=g_{\mu \nu}^{0}(x) d x^{\mu} \otimes d x^{\nu}$ is an arbitrary metric with any signature on the manifold $M_{0}$ and $g^{i}=g_{m_{i} n_{i}}^{i}\left(y_{i}\right) d y_{i}^{m_{i}} \otimes d y_{i}^{n_{i}}$ is a metric on $M_{i}$ satisfying the equation

$$
R_{m_{i} n_{i}}\left[g^{i}\right]=\xi_{i} g_{m_{i} n_{i}}^{i},
$$

$m_{i}, n_{i}=1, \ldots, d_{i} ; \xi_{i}=$ const, $i=1, \ldots, n$. Here $\hat{g}^{i}=p_{i}^{*} g^{i}$ is the pullback of the metric $g^{i}$ to the manifold $M$ by the canonical projection: $p_{i}: M \rightarrow M_{i}, i=0, \ldots, n$. Thus, $\left(M_{i}, g^{i}\right)$ are Einstein spaces, $i=1, \ldots, n$. The functions $\gamma, \phi^{i}: M_{0} \rightarrow \mathbb{R}$ are smooth. We denote $d_{\nu}=\operatorname{dim} M_{\nu} ; \nu=0, \ldots, n ; D=\sum_{\nu=0}^{n} d_{\nu}$. We put any manifold $M_{\nu}, \nu=0, \ldots, n$, to be oriented and connected. Then the volume $d_{i}$-form

$$
\tau_{i} \equiv \sqrt{\left|g^{i}\left(y_{i}\right)\right|} d y_{i}^{1} \wedge \cdots \wedge d y_{i}^{d_{i}},
$$

and signature parameter

$$
\varepsilon(i) \equiv \operatorname{sign}\left(\operatorname{det}\left(g_{m_{i} n_{i}}^{i}\right)\right)= \pm 1
$$

are correctly defined for all $i=1, \ldots, n$.

Let $\Omega=\Omega(n)$ be a set of all non-empty subsets of $\{1, \ldots, n\}$. The number of elements in $\Omega$ is $|\Omega|=2^{n}-1$. For any $I=\left\{i_{1}, \ldots, i_{k}\right\} \in \Omega, i_{1}<\ldots<i_{k}$, we denote

$$
\begin{aligned}
& \tau(I) \equiv \hat{\tau}_{i_{1}} \wedge \cdots \wedge \hat{\tau}_{i_{k}}, \\
& \varepsilon(I) \equiv \varepsilon\left(i_{1}\right) \cdots \varepsilon\left(i_{k}\right), \\
& M_{I} \equiv M_{i_{1}} \times \cdots \times M_{i_{k}}, \\
& d(I) \equiv \sum_{i \in I} d_{i} .
\end{aligned}
$$

Here $\hat{\tau}_{i}=p_{i}^{*} \hat{\tau}_{i}$ is the pullback of the form $\tau_{i}$ to the manifold $M$ by the canonical projection: $p_{i}: M \rightarrow M_{i}, i=1, \ldots, n$. We also put $\tau(\varnothing)=\varepsilon(\varnothing)=1$ and $d(\varnothing)=0$.

For fields of forms we consider the following composite electromagnetic ansatz

$$
F^{a}=\sum_{I \in \Omega_{a, e}} \mathcal{F}^{(a, e, I)}+\sum_{J \in \Omega_{a, m}} \mathcal{F}^{(a, m, J)}
$$

where

$$
\begin{aligned}
& \mathcal{F}^{(a, e, I)}=d \Phi^{(a, e, I)} \wedge \tau(I), \\
& \mathcal{F}^{(a, m, J)}=e^{-2 \lambda_{a}(\varphi)} *\left(d \Phi^{(a, m, J)} \wedge \tau(J)\right)
\end{aligned}
$$

are elementary forms of electric and magnetic types respectively, $a \in \boldsymbol{\Delta}, I \in \Omega_{a, e}, J \in \Omega_{a, m}$ and $\Omega_{a, v} \subset \Omega, v=e, m$. In $(2.7) *=*[g]$ is the Hodge operator on $(M, g)$.

For scalar functions we put

$$
\varphi^{\alpha}=\varphi^{\alpha}(x), \quad \Phi^{s}=\Phi^{s}(x), \quad s \in S .
$$

Thus, $\varphi^{\alpha}$ and $\Phi^{s}$ are functions on $M_{0}$.

Here and below

$$
S=S_{e} \sqcup S_{m}, \quad S_{v}=\sqcup_{a \in \Delta}\{a\} \times\{v\} \times \Omega_{a, v}, \quad v=e, m .
$$


Here and in what follows $\sqcup$ means the union of non-intersecting sets. The set $S$ consists of elements $s=\left(a_{s}, v_{s}, I_{s}\right)$, where $a_{s} \in \boldsymbol{\Delta}$ is color index, $v_{s}=e, m$ is electro-magnetic index and set $I_{s} \in \Omega_{a_{s}, v_{s}}$ describes the location of brane.

Due to (2.6) and (2.7)

$$
d(I)=n_{a}-1, \quad d(J)=D-n_{a}-1
$$

for $I \in \Omega_{a, e}$ and $J \in \Omega_{a, m}$ (i.e. in the electric and magnetic case, respectively).

\subsection{The sigma model}

Let $d_{0} \neq 2$ and

$$
\gamma=\gamma_{0}(\phi) \equiv \frac{1}{2-d_{0}} \sum_{j=1}^{n} d_{j} \phi^{j}
$$

i.e. the generalized harmonic gauge (frame) is used.

Here we put two restrictions on sets of branes that guarantee the block-diagonal form of the energy-momentum tensor and the existence of the sigma-model representation (without additional constraints):

$$
\text { (R1) } d(I \cap J) \leq d(I)-2,
$$

for any $I, J \in \Omega_{a, v}, a \in \boldsymbol{\Delta}, v=e, m$ (here $\left.d(I)=d(J)\right)$ and

$$
\text { (R2) } \quad d(I \cap J) \neq 0 \quad \text { for } \quad d_{0}=1, \quad d(I \cap J) \neq 1 \quad \text { for } \quad d_{0}=3 .
$$

It was proved in [48] that equations of motion for the model (2.1) and the Bianchi identities:

$$
d \mathcal{F}^{s}=0, \quad s \in S_{m},
$$

for fields from (2.3), (2.5)-(2.8), when Restrictions (2.11) and (2.12) are imposed, are equivalent to the equations of motion for the $\sigma$-model governed by the action

$$
\begin{aligned}
S_{\sigma 0}= & \frac{1}{2 \kappa_{0}^{2}} \int d^{d_{0}} x \sqrt{\left|g^{0}\right|}\left\{R\left[g^{0}\right]-\hat{G}_{A B} g^{0 \mu \nu} \partial_{\mu} \sigma^{A} \partial_{\nu} \sigma^{B}\right. \\
& \left.-\sum_{s \in S} \varepsilon_{s} \exp \left(-2 U_{A}^{s} \sigma^{A}\right) g^{0 \mu \nu} \partial_{\mu} \Phi^{s} \partial_{\nu} \Phi^{s}-2 V\right\},
\end{aligned}
$$

where $\left(\sigma^{A}\right)=\left(\phi^{i}, \varphi^{\alpha}\right), k_{0} \neq 0$, the index set $S$ is defined in (2.9),

$$
V=V(\phi)=\Lambda e^{2 \gamma_{0}(\phi)}-\frac{1}{2} \sum_{i=1}^{n} \xi_{i} d_{i} e^{-2 \phi^{i}+2 \gamma_{0}(\phi)}
$$

is the potential,

$$
\left(\hat{G}_{A B}\right)=\left(\begin{array}{cc}
G_{i j} & 0 \\
0 & h_{\alpha \beta}
\end{array}\right)
$$

is the target space metric with

$$
G_{i j}=d_{i} \delta_{i j}+\frac{d_{i} d_{j}}{d_{0}-2}
$$


and co-vectors

$$
U_{A}^{s}=U_{A}^{s} \sigma^{A}=\sum_{i \in I_{s}} d_{i} \phi^{i}-\chi_{s} \lambda_{a_{s}}(\varphi), \quad\left(U_{A}^{s}\right)=\left(d_{i} \delta_{i I_{s}},-\chi_{s} \lambda_{a_{s} \alpha}\right), \quad s=\left(a_{s}, v_{s}, I_{s}\right) .
$$

Here $\chi_{s}=+1$ for $v_{s}=e$ and $\chi_{s}=-1$ for $v_{s}=m$;

$$
\delta_{i I}=\sum_{j \in I} \delta_{i j}
$$

is an indicator of $i$ belonging to $I: \delta_{i I}=1$ for $i \in I$ and $\delta_{i I}=0$ otherwise; and

$$
\varepsilon_{s}=(-\varepsilon[g])^{\left(1-\chi_{s}\right) / 2} \varepsilon\left(I_{s}\right) \theta_{a_{s}}, \quad s \in S, \quad \varepsilon[g] \equiv \operatorname{sign} \operatorname{det}\left(g_{M N}\right) .
$$

More explicitly (2.16) reads

$$
\varepsilon_{s}=\varepsilon\left(I_{s}\right) \theta_{a_{s}} \quad \text { for } \quad v_{s}=e, \quad \varepsilon_{s}=-\varepsilon[g] \varepsilon\left(I_{s}\right) \theta_{a_{s}} \quad \text { for } \quad v_{s}=m .
$$

For finite internal space volumes $V_{i}$ (e.g. compact $M_{i}$ ) and electric $p$-branes (i.e. all $\Omega_{a, m}=\varnothing$ ) the action (2.13) coincides with the action (2.1) when $\kappa^{2}=\kappa_{0}^{2} \prod_{i=1}^{n} V_{i}$.

\section{Solutions governed by harmonic functions}

\subsection{Solutions with block-orthogonal set of $U^{s}$ and Ricci-flat factor-spaces}

Here we consider a special class of solutions to equations of motion governed by several harmonic functions when all factor spaces are Ricci-flat and the cosmological constant is zero, i.e. $\xi_{i}=$ $\Lambda=0, i=1, \ldots, n$. In certain situations these solutions describe extremal black branes charged by fields of forms.

The solutions crucially depend upon scalar products of $U^{s}$-vectors $\left(U^{s}, U^{s^{\prime}}\right) ; s, s^{\prime} \in S$, where

$$
\left(U, U^{\prime}\right)=\hat{G}^{A B} U_{A} U_{B}^{\prime},
$$

for $U=\left(U_{A}\right), U^{\prime}=\left(U_{A}^{\prime}\right) \in \mathbb{R}^{N}, N=n+l$ and

$$
\left(\hat{G}^{A B}\right)=\left(\begin{array}{cc}
G^{i j} & 0 \\
0 & h^{\alpha \beta}
\end{array}\right)
$$

is the inverse matrix to the matrix (2.14). Here as in [67] we have

$$
G^{i j}=\frac{\delta^{i j}}{d_{i}}+\frac{1}{2-D}, \quad i, j=1, \ldots, n
$$

The scalar products (3.1) for vectors $U^{s}$ were calculated in [48] and are given by

$$
\left(U^{s}, U^{s^{\prime}}\right)=d\left(I_{s} \cap I_{s^{\prime}}\right)+\frac{d\left(I_{s}\right) d\left(I_{s^{\prime}}\right)}{2-D}+\chi_{s} \chi_{s^{\prime}} \lambda_{a_{s} \alpha} \lambda_{a_{s^{\prime}} \beta} h^{\alpha \beta},
$$

where $\left(h^{\alpha \beta}\right)=\left(h_{\alpha \beta}\right)^{-1}$, and $s=\left(a_{s}, v_{s}, I_{s}\right), s^{\prime}=\left(a_{s^{\prime}}, v_{s^{\prime}}, I_{s^{\prime}}\right)$ belong to $S$. This relation is a very important one since it encodes brane data (e.g. intersections) via the scalar products of $U$-vectors.

Let

$$
S=S_{1} \sqcup \cdots \sqcup S_{k}, \quad S_{i} \neq \varnothing, \quad i=1, \ldots, k,
$$


and

$$
\left(U^{s}, U^{s^{\prime}}\right)=0
$$

for all $s \in S_{i}, s^{\prime} \in S_{j}, i \neq j ; i, j=1, \ldots, k$. Relation (3.3) means that the set $S$ is a union of $k$ non-intersecting (non-empty) subsets $S_{1}, \ldots, S_{k}$. According to (3.4) the set of vectors $\left(U^{s}, s \in S\right)$ has a block-orthogonal structure with respect to the scalar product (3.1), i.e. it splits into $k$ mutually orthogonal blocks $\left(U^{s}, s \in S_{i}\right), i=1, \ldots, k$.

Here we consider exact solutions in the model (2.1), when vectors $\left(U^{s}, s \in S\right)$ obey the blockorthogonal decomposition (3.3), (3.4) with scalar products defined in (3.2) [37]. These solutions were obtained from the corresponding solutions to the $\sigma$-model equations of motion [37].

Proposition 3.1. Let $\left(M_{0}, g^{0}\right)$ be Ricci-flat: $R_{\mu \nu}\left[g^{0}\right]=0$. Then the field configuration

$$
g^{0}, \quad \sigma^{A}=\sum_{s \in S} \varepsilon_{s} U^{s A} \nu_{s}^{2} \ln H_{s}, \quad \Phi^{s}=\frac{\nu_{s}}{H_{s}}, \quad s \in S,
$$

satisfies the field equations corresponding to the action (2.13) with $V=0$ if the real numbers $\nu_{s}$ obey the relations

$$
\sum_{s^{\prime} \in S}\left(U^{s}, U^{s^{\prime}}\right) \varepsilon_{s^{\prime}} \nu_{s^{\prime}}^{2}=-1, \quad s \in S
$$

the functions $H_{s}>0$ are harmonic, i.e. $\Delta\left[g^{0}\right] H_{s}=0, s \in S$, and $H_{s}$ are coinciding inside blocks: $H_{s}=H_{s^{\prime}}$ for $s, s^{\prime} \in S_{i}, i=1, \ldots, k$.

Using the sigma-model solution from Proposition 3.1 and the relations for contra-variant components [48]:

$$
U^{s i}=\delta_{i I_{s}}-\frac{d\left(I_{s}\right)}{D-2}, \quad U^{s \alpha}=-\chi_{s} \lambda_{a_{s}}^{\alpha}, \quad s=\left(a_{s}, v_{s}, I_{s}\right),
$$

we get [37]:

$$
\begin{aligned}
& g=\left(\prod_{s \in S} H_{s}^{2 d\left(I_{s}\right) \varepsilon_{s} \nu_{s}^{2}}\right)^{1 /(2-D)}\left\{\hat{g}^{0}+\sum_{i=1}^{n}\left(\prod_{s \in S} H_{s}^{2 \varepsilon_{s} \nu_{s}^{2} \delta_{i I_{s}}}\right) \hat{g}^{i}\right\}, \\
& \varphi^{\alpha}=-\sum_{s \in S} \lambda_{a_{s}}^{\alpha} \chi_{s} \varepsilon_{s} \nu_{s}^{2} \ln H_{s}, \\
& F^{a}=\sum_{s \in S} \mathcal{F}^{s} \delta_{a_{s}}^{a},
\end{aligned}
$$

where $i=1, \ldots, n, \alpha=1, \ldots, l, a \in \boldsymbol{\Delta}$ and

$$
\begin{aligned}
& \mathcal{F}^{s}=\nu_{s} d H_{s}^{-1} \wedge \tau\left(I_{s}\right) \text { for } v_{s}=e, \\
& \mathcal{F}^{s}=\nu_{s}\left(*_{0} d H_{s}\right) \wedge \tau\left(\bar{I}_{s}\right) \text { for } v_{s}=m,
\end{aligned}
$$

$H_{s}$ are harmonic functions on $\left(M_{0}, g^{0}\right)$ which coincide inside blocks (i.e. $H_{s}=H_{s^{\prime}}$ for $s, s^{\prime} \in$ $\left.S_{i}, i=1, \ldots, k\right)$ and the relations (3.5) on the parameters $\nu_{s}$ are imposed. Here the matrix $\left(\left(U^{s}, U^{s^{\prime}}\right)\right)$ and parameters $\varepsilon_{s}, s \in S$, are defined in (3.2) and (2.16), respectively; $\lambda_{a}^{\alpha}=h^{\alpha \beta} \lambda_{a \beta}$, $*_{0}=*\left[g^{0}\right]$ is the Hodge operator on $\left(M_{0}, g^{0}\right)$ and

$$
\bar{I}=\{1, \ldots, n\} \backslash I
$$

is the dual set. (In (3.10) we redefined the sign of $\nu_{s}$-parameter.) 


\subsection{Solutions related to non-singular KM algebras}

Now we study the solutions (3.6)-(3.10) in more detail and show that some of them may be related to non-singular KM algebras. We put

$$
K_{s} \equiv\left(U^{s}, U^{s}\right) \neq 0
$$

for all $s \in S$ and introduce the matrix $A=\left(A_{s s^{\prime}}\right)$ :

$$
A_{s s^{\prime}} \equiv \frac{2\left(U^{s}, U^{s^{\prime}}\right)}{\left(U^{s^{\prime}}, U^{s^{\prime}}\right)}, \quad s, s^{\prime} \in S
$$

Here some ordering in $S$ is assumed.

Using this definition and (3.2) we obtain the intersection rules [47]

$$
d\left(I_{s} \cap I_{s^{\prime}}\right)=\Delta\left(s, s^{\prime}\right)+\frac{1}{2} K_{s^{\prime}} A_{s s^{\prime}}
$$

where $s \neq s^{\prime}$, and

$$
\Delta\left(s, s^{\prime}\right)=\frac{d\left(I_{s}\right) d\left(I_{s^{\prime}}\right)}{D-2}-\chi_{s} \chi_{s^{\prime}} \lambda_{a_{s} \alpha} \lambda_{a_{s^{\prime}} \beta} h^{\alpha \beta}
$$

defines the so-called "orthogonal" intersection rules [48] (see also $[53,54,55]$ for $d_{i}=1$ ).

In $D=11$ and $D=10(I I A$ and $I I B)$ supergravity models all $K_{s}=2$ and hence the intersection rules (3.12) in this case have a simpler form [47]:

$$
d\left(I_{s} \cap I_{s^{\prime}}\right)=\Delta\left(s, s^{\prime}\right)+A_{s s^{\prime}}
$$

where $s \neq s^{\prime}$, implying $A_{s s^{\prime}}=A_{s^{\prime} s}$. The corresponding KM algebra is simply-laced in this case.

For $\operatorname{det} A \neq 0$ relation (3.5) may be rewritten in the equivalent form

$$
-\varepsilon_{s} \nu_{s}^{2}\left(U^{s}, U^{s}\right)=2 \sum_{s^{\prime} \in S} A^{s s^{\prime}} \equiv b_{s},
$$

where $s \in S$ and $\left(A^{s s^{\prime}}\right)=A^{-1}$. Thus, equation (3.5) may be resolved in terms of $\nu_{s}$ for certain $\varepsilon_{s}= \pm 1, s \in S$. We note that due to (3.4) the matrix $A$ has a block-diagonal structure and, hence, for any $i$-th block the set of parameters $\left(\nu_{s}, s \in S_{i}\right)$ depends upon the matrix inverse to the matrix $\left(A_{s s^{\prime}} ; s, s^{\prime} \in S_{i}\right)$.

Now we consider one-block case when the brane intersections are related to some non-singular KM algebras.

Finite-dimensional Lie algebras [38]. Let $A$ be a Cartan matrix of a simple finitedimensional Lie algebra. In this case $A_{s s^{\prime}} \in\{0,-1,-2,-3\}, s \neq s^{\prime}$. The elements of inverse matrix $A^{-1}$ are positive (see Chapter 7 in [4]) and hence we get from (3.15) the same signature relation as in the orthogonal case [48]:

$$
\varepsilon_{s}\left(U^{s}, U^{s}\right)<0, \quad s \in S .
$$

When all $\left(U^{s}, U^{s}\right)>0$ we get $\varepsilon_{s}<0, s \in S$.

Moreover, all $b_{s}$ are natural numbers:

$$
b_{s}=n_{s} \in \mathbb{N}, \quad s \in S .
$$

The integers $n_{s}$ coincide with the components of the twice dual Weyl vector in the basis of simple co-roots (see Chather 3.1.7 in [4]). 
Hyperbolic KM algebras. Let $A$ be a generalized Cartan matrix corresponding to a simple hyperbolic KM algebra.

For the hyperbolic algebras the following relations are satisfied

$$
\varepsilon_{s}\left(U^{s}, U^{s}\right)>0
$$

since all $b_{s}$ are negative in the hyperbolic case [31]:

$$
b_{s}<0, \quad \text { where } \quad s \in S \text {. }
$$

For $\left(U^{s}, U^{s}\right)>0$ we get $\varepsilon_{s}>0, s \in S$. If $\theta_{a_{s}}>0$ for all $s \in S$, then

$$
\varepsilon\left(I_{s}\right)=1 \quad \text { for } v_{s}=e, \quad \varepsilon\left(I_{s}\right)=-\varepsilon[g] \text { for } v_{s}=m .
$$

For a metric $g$ of pseudo-Euclidean signature all $\varepsilon\left(I_{s}\right)=1$ and, hence, all branes are Euclidean or should contain even number of time directions: $2,4, \ldots$ For $\varepsilon[g]=1$ only magnetic branes may be pseudo-Euclidean.

Remark 3.1. The inequalities (3.18) guarantee the existence of a principal (real) $s o(1,2)$ subalgebra for any hyperbolic Kac-Moody algebra [68, 31]. Similarly the inequalities (3.16) imply the existence of a principal so(3) subalgebra for any finite dimensional (semi-)simple Lie algebra. It was shown in [31] that this property is not just restricted to hyperbolic algebras, but holds for a wider class of Lorentzian algebras obeying the inequalities $b_{s} \leq 0$ for all $s$.

Example 3.1. $\mathcal{F}_{3}=\boldsymbol{A E}_{\mathbf{3}}$ algebra [39]. Now we consider an example of the solution corresponding to the hyperbolic KM algebra $\mathcal{F}_{3}$ with the Cartan matrix

$$
A=\left(\begin{array}{ccc}
2 & -2 & 0 \\
-2 & 2 & -1 \\
0 & -1 & 2
\end{array}\right)
$$

$\mathcal{F}_{3}$ contains $\mathbf{A}_{\mathbf{1}}^{(\mathbf{1})}$ affine subalgebra (it corresponds to the Geroch group) and $\mathbf{A}_{\mathbf{2}}$ subalgebra. There exists an example of the solution with the $A$-matrix (3.19) for 11-dimensional model governed by the action

$$
S=\int d^{11} z \sqrt{|g|}\left\{R[g]-\frac{1}{4 !}\left(F^{4}\right)^{2}-\frac{1}{4 !}\left(F^{4 *}\right)^{2}\right\}
$$

where $\operatorname{rank} F^{4}=\operatorname{rank} F^{4 *}=4$. Here $\boldsymbol{\Delta}=\{4,4 *\}$. We consider a configuration with two magnetic 5-branes corresponding to the form $F^{4}$ and one electric 2-brane corresponding to the form $F^{4 *}$. We denote $S=\left\{s_{1}, s_{2}, s_{3}\right\}, a_{s_{1}}=a_{s_{3}}=4, a_{s_{2}}=4 *$ and $v_{s_{1}}=v_{s_{3}}=m, v_{s_{2}}=e$, where $d\left(I_{s_{1}}\right)=d\left(I_{s_{3}}\right)=6$ and $d\left(I_{s_{2}}\right)=3$.

The intersection rules (3.12) read

$$
d\left(I_{s_{1}} \cap I_{s_{2}}\right)=0, \quad d\left(I_{s_{2}} \cap I_{s_{3}}\right)=1, \quad d\left(I_{s_{1}} \cap I_{s_{3}}\right)=4 .
$$

For the manifold $(2.2)$ we put $n=5$ and $d_{1}=2, d_{2}=4, d_{3}=d_{4}=1, d_{5}=2$. The corresponding brane sets are the following: $I_{s_{1}}=\{1,2\}, I_{s_{2}}=\{4,5\}, I_{s_{3}}=\{2,3,4\}$.

The solution reads

$$
\begin{aligned}
& g=H^{-12}\left\{-d t \otimes d t+H^{9} \hat{g}^{1}+H^{13} \hat{g}^{2}+H^{4} \hat{g}^{3}+H^{14} \hat{g}^{4}+H^{10} \hat{g}^{5}\right\} \\
& F^{4}=\frac{d H}{d t}\left\{\nu_{s_{1}} \hat{\tau}_{3} \wedge \hat{\tau}_{4} \wedge \hat{\tau}_{5}+\nu_{s_{3}} \hat{\tau}_{1} \wedge \hat{\tau}_{5}\right\}
\end{aligned}
$$




$$
F^{4 *}=\frac{d H}{d t} \frac{\nu_{s_{2}}}{H^{2}} d t \wedge \hat{\tau}_{4} \wedge \hat{\tau}_{5},
$$

where $\nu_{s_{1}}^{2}=\frac{9}{2}, \nu_{s_{2}}^{2}=5$ and $\nu_{s_{3}}^{2}=2($ see $(3.15))$.

All metrics $g^{i}$ are Ricci-flat $(i=1, \ldots, 5)$ and have Euclidean signatures (this agrees with relations (3.17) and (2.16)), and $H=h t+h_{0}>0$, where $h, h_{0}$ are constants. The metric (3.20) may be also rewritten using the synchronous time variable $t_{s}$

$$
g=-d t_{s} \otimes d t_{s}+f^{3 / 5} \hat{g}^{1}+f^{-1 / 5} \hat{g}^{2}+f^{8 / 5} \hat{g}^{3}+f^{-2 / 5} \hat{g}^{4}+f^{2 / 5} \hat{g}^{5},
$$

where $f=5 h t_{s}=H^{-5}>0, h>0$ and $t_{s}>0$. The metric describes the power-law "inflation" in $D=11$. It is singular for $t_{s} \rightarrow+0$.

In the next example we consider a chain of the so-called $B_{D}$-models $(D \geq 11)$ suggested in [47]. For $D=11$ the $B_{D}$-model coincides with the truncated (i.e. without Chern-Simons term) bosonic sector of $D=11$ supergravity [27] which is related to $M$-theory. For $D=12$ it coincides with truncated 12-dimensional model from [69] which may be related to $F$-theory [70].

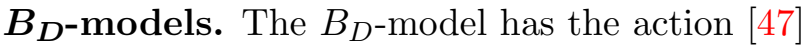

$$
S_{D}=\int d^{D} z \sqrt{|g|}\left\{R[g]+g^{M N} \partial_{M} \vec{\varphi} \partial_{N} \vec{\varphi}-\sum_{a=4}^{D-7} \frac{1}{a !} \exp \left[2 \vec{\lambda}_{a} \vec{\varphi}\right]\left(F^{a}\right)^{2}\right\},
$$

where $\vec{\varphi}=\left(\varphi_{\overrightarrow{\lambda_{a}}}^{1}, \ldots, \varphi^{l}\right) \in \mathbb{R}^{l}, \vec{\lambda}_{a}=\left(\lambda_{a 1}, \ldots, \lambda_{a l}\right) \in \mathbb{R}^{l}, l=D-11, \operatorname{rank} F^{a}=a, a=4, \ldots, D-7$. Here vectors $\vec{\lambda}_{a}$ satisfy the relations

$$
\vec{\lambda}_{a} \vec{\lambda}_{b}=N(a, b)-\frac{(a-1)(b-1)}{D-2}=\Lambda_{a b}, \quad N(a, b)=\min (a, b)-3,
$$

$a, b=4, \ldots, D-7$ and $\vec{\lambda}_{D-7}=-2 \vec{\lambda}_{4}$. For $D>11$ vectors $\vec{\lambda}_{4}, \ldots, \vec{\lambda}_{D-8}$ are linearly independent. (It may be verified that matrix $\left(\Lambda_{a b}\right)$ is positive definite and hence the set of vectors obeying (3.22) does exist.)

The model (3.21) contains $l$ scalar fields with a negative kinetic term (i.e. $h_{\alpha \beta}=-\delta_{\alpha \beta}$ in $(2.1)$ ) coupled to several forms (the number of forms is $(l+1))$.

For the brane worldvolumes we have the following dimensions (see (2.10))

$$
\begin{array}{ll}
d(I)=3, \ldots, D-8, & I \in \Omega_{a, e}, \\
d(I)=D-5, \ldots, 6, & I \in \Omega_{a, m} .
\end{array}
$$

Thus, there are $(l+1)$ electric and $(l+1)$ magnetic $p$-branes, $p=d(I)-1$. In $B_{D}$-model all $K_{s}=2$.

Example 3.2. $H_{2}\left(q_{1}, q_{2}\right)$ algebra [37]. Let

$$
A=\left(\begin{array}{cc}
2 & -q_{1} \\
-q_{2} & 2
\end{array}\right), \quad q_{1} q_{2}>4
$$

$q_{1}, q_{2} \in \mathbb{N}$. This is the Cartan matrix for the hyperbolic KM algebra $H_{2}\left(q_{1}, q_{2}\right)$ [3]. From (3.15) we get

$$
\varepsilon_{s} \nu_{s}^{2}\left(U^{s}, U^{s}\right)\left(q_{1} q_{2}-4\right)=2 q_{s}+4,
$$

$s \in\{1,2\}=S$. An example of the $H_{2}(q, q)$-solution for $B_{D}$-model with two electric $p$-branes $(p=$ $\left.d_{1}, d_{2}\right)$, corresponding to $F^{a}$ and $F^{b}$ fields and intersecting on time manifold, is the following [37]:

$$
g=H^{-2 /(q-2)} \hat{g}^{0}-H^{2 /(q-2)} d t \otimes d t+\hat{g}^{1}+\hat{g}^{2},
$$




$$
\begin{aligned}
& F^{a}=\nu_{1} d H^{-1} \wedge d t \wedge \hat{\tau}_{1}, \\
& F^{b}=\nu_{2} d H^{-1} \wedge d t \wedge \hat{\tau}_{2}, \\
& \vec{\varphi}=\left(\vec{\lambda}_{a}+\vec{\lambda}_{b}\right)(q-2)^{-1} \ln H,
\end{aligned}
$$

where $d_{0}=3, d_{1}=a-2, a=q+4, a<b, d_{2}=b-2, D=a+b$, and $\nu_{1}^{2}=\nu_{2}^{2}=(q-2)^{-1}$. The signature restrictions are : $\varepsilon(1)=\varepsilon(2)=-1$. Thus, the space-time $(M, g)$ should contain at least three time directions. The minimal $D$ is 15 . For $D=15$ we get $a=7, b=8, d_{1}=5$, $d_{2}=6$ and $q=3$. (Here we have eliminated a typo in a sign of scalar fields that was originally in [37].)

Remark 3.2. We note that affine KM algebras (with $\operatorname{det} A=0$ ) do not appear in the solutions (3.6)-(3.10). Indeed, any affine Cartan matrix satisfy the relations

$$
\sum_{s \in S} a_{s} A_{s s^{\prime}}=0
$$

with $a_{s}>0$ called Coxeter labels [4], $s \in S$. This relation makes impossible the existence of the solution to equation (3.5) (see (3.11)).

Generalized Majumdar-Papapetrou solutions. Now we return to a "multi-block" solution (3.6)-(3.10). Let $M_{0}=\mathbb{R}^{d_{0}}, d_{0}>2, g^{0}=\delta_{\mu \nu} d x^{\mu} \otimes d x^{\nu}, d_{1}=1$ and $g^{1}=-d t \otimes d t$. For

$$
H_{s}=1+\sum_{b \in X_{s}} \frac{q_{s b}}{|x-b|^{d_{0}-2}}
$$

where $X_{s}$ is finite non-empty subset $X_{s} \subset M_{0}, s \in S$, all $q_{s b}>0$, and $X_{s}=X_{s^{\prime}}, q_{s b}=q_{s^{\prime} b}$ for $b \in X_{s}=X_{s^{\prime}}, s, s^{\prime} \in S_{j}, j=1, \ldots, k$. The harmonic functions (3.23) are defined in domain $M_{0} \backslash X, X=\bigcup_{s \in S} X_{s}$, and generate the solutions (3.6)-(3.10).

Denote $S(b) \equiv\left\{s \in S \mid \quad b \in X_{s}\right\}, b \in X$. (In the one-block case, when $k=1$, all $X_{s}=X$ and $S(b)=S$.) We have a horizon at point $b$ w.r.t. time $t$, when $x \rightarrow b \in X$, if and only if

$$
\xi_{1}(b) \equiv \sum_{s \in S(b)}\left(-\varepsilon_{s}\right) \nu_{s}^{2} \delta_{1 I_{s}}-\frac{1}{d_{0}-2} \geq 0 .
$$

This relation follows just from the requirement of the infinite propagation time of light to $b \in X$.

Majumdar-Papapetrou solution. Recall that the well-known 4-dimensional MajumdarPapapetrou (MP) solution [71] corresponding to the Lie algebra $\mathbf{A}_{\mathbf{1}}$ in our notations reads

$$
\begin{aligned}
& g=H^{2} \hat{g}^{0}-H^{-2} d t \otimes d t, \\
& F=\nu d H^{-1} \wedge d t,
\end{aligned}
$$

where $\nu^{2}=2, g^{0}=\sum_{i=1}^{3} d x^{i} \otimes d x^{i}$ and $H$ is a harmonic function. We have one electric 0-brane (point) "attached" to the time manifold; $d\left(I_{s}\right)=1, \varepsilon_{s}=-1$ and $\left(U^{s}, U^{s}\right)=1 / 2$. In this case (e.g. for the extremal Reissner-Nordström black hole) we get $\xi_{1}(b)=1, b \in X$. Points $b$ are the points of (regular) horizon.

For certain examples of finite-dimensional semisimple Lie algebras (e.g. for $\mathbf{A}_{\mathbf{1}} \oplus \cdots \oplus \mathbf{A}_{\mathbf{1}}$, $\mathbf{A}_{2}$ etc.) the poles $b$ in $H_{s}$ correspond to (regular) horizons and the solution under consideration describes a collection of $k$ blocks of extremal charged black branes (in equilibrium) [37].

Hyperbolic KM algebras. Let us consider a generalized one-block $(k=1)$ MP solution corresponding to a hyperbolic KM algebra such that $\left(U^{s}, U^{s}\right)>0$ for all $s \in S$. In this case all $\varepsilon_{s}>0, s \in S$, and hence $\xi_{1}(b)<0$. Thus, any point $b \in X$ is not a point of the horizon. (It may be checked using the analysis carried out in [37] that any non-exceptional point $b$ is a singular one). As a consequence, the generalized MP solution corresponding to any hyperbolic KM algebra does not describe a collection of extremal charged black branes (in equilibrium) when all $\left(U^{s}, U^{s}\right)>0$. 


\subsection{Toda-like solutions}

\subsubsection{Toda-like Lagrangian}

Action (2.13) may be also written in the form

$$
S_{\sigma 0}=\frac{1}{2 \kappa_{0}^{2}} \int d^{d_{0}} x \sqrt{\left|g^{0}\right|}\left\{R\left[g^{0}\right]-\mathcal{G}_{\hat{A} \hat{B}}(X) g^{0 \mu \nu} \partial_{\mu} X^{\hat{A}} \partial_{\nu} X^{\hat{B}}-2 V\right\},
$$

where $X=\left(X^{\hat{A}}\right)=\left(\phi^{i}, \varphi^{\alpha}, \Phi^{s}\right) \in R^{N}$, and the minisupermetric $\mathcal{G}=\mathcal{G}_{\hat{A} \hat{B}}(X) d X^{\hat{A}} \otimes d X^{\hat{B}}$ on the minisuperspace $\mathcal{M}=\mathbf{R}^{N}, N=n+l+|S|(|S|$ is the number of elements in $S)$ is defined by the relation

$$
\left(\mathcal{G}_{\hat{A} \hat{B}}(X)\right)=\left(\begin{array}{ccc}
G_{i j} & 0 & 0 \\
0 & h_{\alpha \beta} & 0 \\
0 & 0 & \varepsilon_{s} \exp \left(-2 U^{s}(\sigma)\right) \delta_{s s^{\prime}}
\end{array}\right) .
$$

Here we consider exact solutions to field equations corresponding to the action (3.24)

$$
\begin{aligned}
& R_{\mu \nu}\left[g^{0}\right]=\mathcal{G}_{\hat{A} \hat{B}}(X) \partial_{\mu} X^{\hat{A}} \partial_{\nu} X^{\hat{B}}+\frac{2 V}{d_{0}-2} g_{\mu \nu}^{0}, \\
& \frac{1}{\sqrt{\left|g^{0}\right|}} \partial_{\mu}\left[\sqrt{\left|g^{0}\right|} \mathcal{G}_{\hat{C} \hat{B}}(X) g^{0 \mu \nu} \partial_{\nu} X^{\hat{B}}\right]-\frac{1}{2} \mathcal{G}_{\hat{A} \hat{B}, \hat{C}}(X) g^{0, \mu \nu} \partial_{\mu} X^{\hat{A}} \partial_{\nu} X^{\hat{B}}=V_{, \hat{C}},
\end{aligned}
$$

where $s \in S$. Here $V_{, \hat{C}}=\partial V / \partial X^{\hat{C}}$.

We put

$$
X^{\hat{A}}(x)=F^{\hat{A}}(H(x)),
$$

where $F:\left(u_{-}, u_{+}\right) \rightarrow \mathbb{R}^{N}$ is a smooth function, $H: M_{0} \rightarrow \mathbb{R}$ is a harmonic function on $M_{0}$ (i.e. $\left.\Delta\left[g^{0}\right] H=0\right)$, satisfying $u_{-}<H(x)<u_{+}$for all $x \in M_{0}$. We take all factor spaces as Ricci-flat and the cosmological constant is set to zero, i.e. the relations $\xi_{i}=0$ and $\Lambda=0$ are satisfied.

In this case the potential is zero : $V=0$. It may be verified that the field equations (3.26) and (3.27) are satisfied identically if $F=F(u)$ obeys the Lagrange equations for the Lagrangian

$$
L=\frac{1}{2} \mathcal{G}_{\hat{A} \hat{B}}(F) \dot{F}^{\hat{A}} \dot{F}^{\hat{B}}
$$

with the zero-energy constraint

$$
E=\frac{1}{2} \mathcal{G}_{\hat{A} \hat{B}}(F) \dot{F}^{\hat{A}} \dot{F}^{\hat{B}}=0 .
$$

This means that $F:\left(u_{-}, u_{+}\right) \rightarrow \mathbb{R}^{N}$ is a null-geodesic map for the minisupermetric $\mathcal{G}$. Thus, we are led to the Lagrange system (3.28) with the minisupermetric $\mathcal{G}$ defined in (3.25).

The problem of integrability will be simplified if we integrate the Lagrange equations corresponding to $\Phi^{s}$ (i.e. the Maxwell-type equations for $s \in S_{e}$ and Bianchi identities for $s \in S_{m}$ ):

$$
\frac{d}{d u}\left(\exp \left(-2 U^{s}(\sigma)\right) \dot{\Phi}^{s}\right)=0 \Longleftrightarrow \dot{\Phi}^{s}=Q_{s} \exp \left(2 U^{s}(\sigma)\right)
$$

where $Q_{s}$ are constants, and $s \in S$. Here $\left(F^{\hat{A}}\right)=\left(\sigma^{A}, \Phi^{s}\right)$. We put $Q_{s} \neq 0$ for all $s \in S$.

For fixed $Q=\left(Q_{s}, s \in S\right)$ the Lagrange equations for the Lagrangian (3.28) corresponding to $\left(\sigma^{A}\right)=\left(\phi^{i}, \varphi^{\alpha}\right)$, when equations (3.30) are substituted, are equivalent to the Lagrange equations for the Lagrangian

$$
L_{Q}=\frac{1}{2} \hat{G}_{A B} \dot{\sigma}^{A} \dot{\sigma}^{B}-V_{Q},
$$


where

$$
V_{Q}=\frac{1}{2} \sum_{s \in S} \varepsilon_{s} Q_{s}^{2} \exp \left[2 U^{s}(\sigma)\right]
$$

the matrix $\left(\hat{G}_{A B}\right)$ is defined in (2.14). The zero-energy constraint (3.29) reads

$$
E_{Q}=\frac{1}{2} \hat{G}_{A B} \dot{\sigma}^{A} \dot{\sigma}^{B}+V_{Q}=0 .
$$

\subsubsection{The solutions}

Here, as above we are interested in exact solutions for a special case when $K_{s}=\left(U^{s}, U^{s}\right) \neq 0$, for all $s \in S$, and the generalized Cartan matrix (3.11) is non-degenerate. It follows from the non-degeneracy of the matrix (3.11) that vectors $U^{s}, s \in S$, are linearly independent. Hence, the number of vectors $U^{s}$ should not exceed the dimension of $\mathbb{R}^{n+l}$, i.e. $|S| \leq n+l$.

The exact solutions were obtained in [40] and are

$$
\begin{aligned}
& g=\left(\prod_{s \in S} f_{s}^{2 d\left(I_{s}\right) h_{s} /(D-2)}\right)\left\{\exp \left(2 c^{0} H+2 \bar{c}^{0}\right) \hat{g}^{0}\right. \\
&\left.+\sum_{i=1}^{n}\left(\prod_{s \in S} f_{s}^{-2 h_{s} \delta_{i I_{s}}}\right) \exp \left(2 c^{i} H+2 \bar{c}^{i}\right) \hat{g}^{i}\right\}, \\
& \exp \left(\varphi^{\alpha}\right)=\left(\prod_{s \in S} f_{s}^{h_{s} \chi_{s} \lambda_{a_{s}}^{\alpha}}\right) \exp \left(c^{\alpha} H+\bar{c}^{\alpha}\right), \quad \alpha=1, \ldots, l,
\end{aligned}
$$

and $F^{a}=\sum_{s \in S} \mathcal{F}^{s} \delta_{a_{s}}^{a}$ with

$$
\begin{aligned}
& \mathcal{F}^{s}=Q_{s}\left(\prod_{s^{\prime} \in S} f_{s^{\prime}}^{-A_{s s^{\prime}}}\right) d H \wedge \tau\left(I_{s}\right), \quad s \in S_{e}, \\
& \mathcal{F}^{s}=Q_{s}\left(*_{0} d H\right) \wedge \tau\left(\bar{I}_{s}\right), \quad s \in S_{m},
\end{aligned}
$$

where $*_{0}=*\left[g^{0}\right]$ is the Hodge operator on $\left(M_{0}, g^{0}\right)$. Here

$$
f_{s}=f_{s}(H)=\exp \left(-q^{s}(H)\right),
$$

where $q^{s}(u)$ is a solution to the Toda-like equations

$$
\ddot{q}^{s}=-B_{s} \exp \left(\sum_{s^{\prime} \in S} A_{s s^{\prime}} q^{s^{\prime}}\right)
$$

with $B_{s}=K_{s} \varepsilon_{s} Q_{s}^{2}, s \in S$, and $H=H(x)\left(x \in M_{0}\right)$ is a harmonic function on $\left(M_{0}, g^{0}\right)$. Vectors $c=\left(c^{A}\right)$ and $\bar{c}=\left(\bar{c}^{A}\right)$ satisfy the linear constraints

$$
U^{s}(c)=\sum_{i \in I_{s}} d_{i} c^{i}-\chi_{s} \lambda_{a_{s} \alpha} c^{\alpha}=0, \quad U^{s}(\bar{c})=0, \quad s \in S,
$$

and

$$
c^{0}=\frac{1}{2-d_{0}} \sum_{j=1}^{n} d_{j} c^{j}, \quad \bar{c}^{0}=\frac{1}{2-d_{0}} \sum_{j=1}^{n} d_{j} \bar{c}^{j} .
$$


The zero-energy constraint reads

$$
2 E_{T}+h_{\alpha \beta} c^{\alpha} c^{\beta}+\sum_{i=1}^{n} d_{i}\left(c^{i}\right)^{2}+\frac{1}{d_{0}-2}\left(\sum_{i=1}^{n} d_{i} c^{i}\right)^{2}=0
$$

where

$$
E_{T}=\frac{1}{4} \sum_{s, s^{\prime} \in S} h_{s} A_{s s^{\prime}} \dot{q} \dot{q^{s^{\prime}}}+\sum_{s \in S} A_{s} \exp \left(\sum_{s^{\prime} \in S} A_{s s^{\prime}} q^{s^{\prime}}\right)
$$

is an integration constant (energy) for the solutions from (3.37) and $A_{s}=\frac{1}{2} \varepsilon_{s} Q_{s}^{2}$.

We note that equations (3.37) correspond to the Lagrangian

$$
L_{T}=\frac{1}{4} \sum_{s, s^{\prime} \in S} h_{s} A_{s s^{\prime}} \dot{q}^{s} q^{\dot{s}^{\prime}}-\sum_{s \in S} A_{s} \exp \left(\sum_{s^{\prime} \in S} A_{s s^{\prime}} q^{s^{\prime}}\right)
$$

where $h_{s}=K_{s}^{-1}$.

Thus, the solution is given by relations (3.34)-(3.36) with the functions $q^{s}$ being defined in (3.37) and with relations on the parameters of solutions $c^{A}, \bar{c}^{A}(A=i, \alpha, 0)$, imposed by (3.38), (3.39), (3.40).

\section{Cosmological-type solutions}

Now we consider the case $d_{0}=1, M_{0}=\mathbb{R}$, i.e. we are interested in applications to the sector with dependence on a single variable. We consider the manifold

$$
M=\left(u_{-}, u_{+}\right) \times M_{1} \times \cdots \times M_{n}
$$

with a metric

$$
g=w e^{2 \gamma(u)} d u \otimes d u+\sum_{i=1}^{n} e^{2 \phi^{i}(u)} \hat{g}^{i}
$$

where $w= \pm 1, u$ is a distinguished coordinate which, by convention, will be called "time"; $\left(M_{i}, g^{i}\right)$ are oriented and connected Einstein spaces (see $\left.(2.4)\right), i=1, \ldots, n$. The functions $\gamma, \phi^{i}$ : $\left(u_{-}, u_{+}\right) \rightarrow \mathbb{R}$ are smooth.

Here we adopt the brane ansatz from Section 2 putting $g^{0}=w d u \otimes d u$.

\subsection{Lagrange dynamics}

It follows from Subsection 2.3 that the equations of motion and the Bianchi identities for the field configuration under consideration (with the restrictions from Subsection 2.3 imposed) are equivalent to equations of motion for 1-dimensional $\sigma$-model with the action

$$
S_{\sigma}=\frac{\mu}{2} \int d u \mathcal{N}\left\{G_{i j} \dot{\phi}^{i} \dot{\phi}^{j}+h_{\alpha \beta} \dot{\varphi}^{\alpha} \dot{\varphi}^{\beta}+\sum_{s \in S} \varepsilon_{s} \exp \left[-2 U^{s}(\phi, \varphi)\right]\left(\dot{\Phi}^{s}\right)^{2}-2 \mathcal{N}^{-2} V_{w}(\phi)\right\},
$$

where $\dot{x} \equiv d x / d u$,

$$
V_{w}=-w V=-w \Lambda e^{2 \gamma_{0}(\phi)}+\frac{w}{2} \sum_{i=1}^{n} \xi_{i} d_{i} e^{-2 \phi^{i}+2 \gamma_{0}(\phi)}
$$


is the potential with $\gamma_{0}(\phi) \equiv \sum_{i=1}^{n} d_{i} \phi^{i}$, and $\mathcal{N}=\exp \left(\gamma_{0}-\gamma\right)>0$ is the modified lapse function, $U^{s}=U^{s}(\phi, \varphi)$ are defined in (2.15), $\varepsilon_{s}$ are defined in (2.16) for $s=\left(a_{s}, v_{s}, I_{s}\right) \in S$, and $G_{i j}=d_{i} \delta_{i j}-d_{i} d_{j}$ are components of "pure cosmological" minisupermetric, $i, j=1, \ldots, n[67]$.

In the electric case $\left(\mathcal{F}^{(a, m, I)}=0\right)$ for finite internal space volumes $V_{i}$ the action (4.1) coincides with the action (2.1) if $\mu=-w / \kappa_{0}^{2}, \kappa^{2}=\kappa_{0}^{2} V_{1} \cdots V_{n}$.

Action (4.1) may be also written in the form

$$
S_{\sigma}=\frac{\mu}{2} \int d u \mathcal{N}\left\{\mathcal{G}_{\hat{A} \hat{B}}(X) \dot{X}^{\hat{A}} \dot{X}^{\hat{B}}-2 \mathcal{N}^{-2} V_{w}\right\}
$$

where $X=\left(X^{\hat{A}}\right)=\left(\phi^{i}, \varphi^{\alpha}, \Phi^{s}\right) \in \mathbb{R}^{N}, N=n+l+|S|$, and minisupermetric $\mathcal{G}$ is defined in $(3.25)$.

Scalar products. The minisuperspace metric (3.25) may be also written in the form $\mathcal{G}=$ $\hat{G}+\sum_{s \in S} \varepsilon_{s} e^{-2 U^{s}(\sigma)} d \Phi^{s} \otimes d \Phi^{s}$, where $\sigma=\left(\sigma^{A}\right)=\left(\phi^{i}, \varphi^{\alpha}\right)$,

$$
\hat{G}=\hat{G}_{A B} d \sigma^{A} \otimes d \sigma^{B}=G_{i j} d \phi^{i} \otimes d \phi^{j}+h_{\alpha \beta} d \varphi^{\alpha} \otimes d \varphi^{\beta},
$$

is the truncated minisupermetric and $U^{s}(\sigma)=U_{A}^{s} \sigma^{A}$ is defined in (2.15). The potential (4.2) now reads

$$
V_{w}=(-w \Lambda) e^{2 U^{\Lambda}(\sigma)}+\sum_{j=1}^{n} \frac{w}{2} \xi_{j} d_{j} e^{2 U^{j}(\sigma)},
$$

where

$$
\begin{aligned}
& U^{j}(\sigma)=U_{A}^{j} \sigma^{A}=-\phi^{j}+\gamma_{0}(\phi), \quad\left(U_{A}^{j}\right)=\left(-\delta_{i}^{j}+d_{i}, 0\right), \\
& U^{\Lambda}(\sigma)=U_{A}^{\Lambda} \sigma^{A}=\gamma_{0}(\phi), \quad\left(U_{A}^{\Lambda}\right)=\left(d_{i}, 0\right) .
\end{aligned}
$$

The integrability of the Lagrange system (4.3) crucially depends upon the scalar products of co-vectors $U^{\Lambda}, U^{j}, U^{s}$ (see (3.1)). These products are defined by (3.2) and the following relations [48]

$$
\begin{array}{ll}
\left(U^{i}, U^{j}\right) & =\frac{\delta_{i j}}{d_{j}}-1, \\
\left(U^{i}, U^{\Lambda}\right) & =-1, \quad\left(U^{\Lambda}, U^{\Lambda}\right)=-\frac{D-1}{D-2}, \\
\left(U^{s}, U^{i}\right) & =-\delta_{i I_{s}}, \quad\left(U^{s}, U^{\Lambda}\right)=\frac{d\left(I_{s}\right)}{2-D},
\end{array}
$$

where $s=\left(a_{s}, v_{s}, I_{s}\right) \in S ; i, j=1, \ldots, n$.

Toda-like representation. We put $\gamma=\gamma_{0}(\phi)$, i.e. the harmonic time gauge is considered. Integrating the Lagrange equations corresponding to $\Phi^{s}$ (see (3.30)) we are led to the Lagrangian from (3.31) and the zero-energy constraint (3.33) with the modified potential

$$
V_{Q}=V_{w}+\frac{1}{2} \sum_{s \in S} \varepsilon_{s} Q_{s}^{2} \exp \left[2 U^{s}(\sigma)\right]
$$

where $V_{w}$ is defined in (4.2).

\subsection{Solutions with $\Lambda=0$}

Here we consider solutions with $\Lambda=0$. 


\subsubsection{Solutions with Ricci-flat factor-spaces}

Let all spaces be Ricci-flat, i.e. $\xi_{1}=\cdots=\xi_{n}=0$.

Since $H(u)=u$ is a harmonic function on $\left(M_{0}, g^{0}\right)$ with $g^{0}=w d u \otimes d u$ we get for the metric and scalar fields from (3.34), (3.35) [40]

$$
\begin{aligned}
& g=\left(\prod _ { s \in S } f _ { s } ^ { 2 d ( I _ { s } ) h _ { s } / ( D - 2 ) ) } \left\{\exp \left(2 c^{0} u+2 \bar{c}^{0}\right) w d u \otimes d u\right.\right. \\
&\left.+\sum_{i=1}^{n}\left(\prod_{s \in S} f_{s}^{-2 h_{s} \delta_{i I_{s}}}\right) \exp \left(2 c^{i} u+2 \bar{c}^{i}\right) \hat{g}^{i}\right\}, \\
& \exp \left(\varphi^{\alpha}\right)=\left(\prod_{s \in S} f_{s}^{h_{s} \chi_{s} \lambda_{a_{s}}^{\alpha}}\right) \exp \left(c^{\alpha} u+\bar{c}^{\alpha}\right), \quad \alpha=1, \ldots, l,
\end{aligned}
$$

and $F^{a}=\sum_{s \in S} \delta_{a_{s}}^{a} \mathcal{F}^{s}$ with

$$
\begin{aligned}
& \mathcal{F}^{s}=Q_{s}\left(\prod_{s^{\prime} \in S} f_{s^{\prime}}^{-A_{s s^{\prime}}}\right) d u \wedge \tau\left(I_{s}\right), \quad s \in S_{e}, \\
& \mathcal{F}^{s}=Q_{s} \tau\left(\bar{I}_{s}\right), \quad s \in S_{m},
\end{aligned}
$$

$Q_{s} \neq 0, s \in S$. Here $f_{s}=f_{s}(u)=\exp \left(-q^{s}(u)\right)$ and $q^{s}(u)$ obey Toda-like equations (3.37).

Relations (3.39) and (3.40) take the form

$$
\begin{aligned}
& c^{0}=\sum_{j=1}^{n} d_{j} c^{j}, \quad \bar{c}^{0}=\sum_{j=1}^{n} d_{j} \bar{c}^{j}, \\
& 2 E_{T}+h_{\alpha \beta} c^{\alpha} c^{\beta}+\sum_{i=1}^{n} d_{i}\left(c^{i}\right)^{2}-\left(\sum_{i=1}^{n} d_{i} c^{i}\right)^{2}=0,
\end{aligned}
$$

with $E_{T}$ from (3.41) and all other relations (e.g. constraints (3.38)) are unchanged.

This solution in the special case of an $\mathbf{A}_{\mathbf{m}}$ Toda chain, was obtained earlier in [72] (see also [73]). Some special configurations were considered earlier in [74, 75, 76].

Currently, the cosmological solutions with branes are considered often in a context of $S$ brane terminology [77]. $S$-branes were originally space-like analogues of $D$-branes, see also $[78,79,80,81,45,82,83]$ and references therein.

\subsubsection{Solutions with one curved factor-space}

The cosmological solution with Ricci-flat spaces may be also modified to the following case: $\xi_{1} \neq 0, \xi_{2}=\cdots=\xi_{n}=0$, i.e. one space is curved and others are Ricci-flat and $1 \notin I_{s}, s \in S$, i.e. all "brane" submanifolds do not contain $M_{1}$.

The potential (3.32) is modified for $\xi_{1} \neq 0$ as follows (see (4.7))

$$
V_{Q}=\frac{1}{2} \sum_{s \in S} \varepsilon_{s} Q_{s}^{2} \exp \left[2 U^{s}(\sigma)\right]+\frac{1}{2} w \xi_{1} d_{1} \exp \left[2 U^{1}(\sigma)\right],
$$

where $U^{1}(\sigma)$ is defined in (4.4) $\left(d_{1}>1\right)$.

For the scalar products we get from (4.5) and (4.6)

$$
\left(U^{1}, U^{1}\right)=\frac{1}{d_{1}}-1<0, \quad\left(U^{1}, U^{s}\right)=0
$$

for all $s \in S$. 
The solution in the case under consideration may be obtained by a little modification of the solution from the previous section (using (4.12), relations $U^{1 i}=-\delta_{1}^{i} / d_{1}, U^{1 \alpha}=0$ ) [40]

$$
\begin{aligned}
& g=\left(\prod_{s \in S}\left[f_{s}(u)\right]^{2 d\left(I_{s}\right) h_{s} /(D-2)}\right)\left\{\left[f_{1}(u)\right]^{2 d_{1} /\left(1-d_{1}\right)} \exp \left(2 c^{1} u+2 \bar{c}^{1}\right)\right. \\
& \left.\quad \times\left[w d u \otimes d u+f_{1}^{2}(u) \hat{g}^{1}\right]+\sum_{i=2}^{n}\left(\prod_{s \in S}\left[f_{s}(u)\right]^{-2 h_{s} \delta_{i I_{s}}}\right) \exp \left(2 c^{i} u+2 \bar{c}^{i}\right) \hat{g}^{i}\right\}, \\
& \exp \left(\varphi^{\alpha}\right)=\left(\prod_{s \in S} f_{s}^{h_{s} \chi_{s} \lambda_{a_{s}}^{\alpha}}\right) \exp \left(c^{\alpha} u+\bar{c}^{\alpha}\right), \\
& F^{a}=\sum_{s \in S} \delta_{a_{s}}^{a} \mathcal{F}^{s},
\end{aligned}
$$

with forms $\mathcal{F}^{s}$ defined in (4.10) and (4.11).

Here $f_{s}=f_{s}(u)=\exp \left(-q^{s}(u)\right)$ where $q^{s}(u)$ obey Toda-like equations (3.37) and

$$
f_{1}(u)= \begin{cases}R \sinh \left(\sqrt{C_{1}}\left(u-u_{1}\right)\right), & C_{1}>0, \quad \xi_{1} w>0 \\ R \sin \left(\sqrt{\left|C_{1}\right|}\left(u-u_{1}\right)\right), & C_{1}<0, \quad \xi_{1} w>0 \\ R \cosh \left(\sqrt{C_{1}}\left(u-u_{1}\right)\right), & C_{1}>0, \quad \xi_{1} w<0 \\ \left|\xi_{1}\left(d_{1}-1\right)\right|^{1 / 2}, & C_{1}=0, \quad \xi_{1} w>0\end{cases}
$$

$u_{1}, C_{1}$ are constants and $R=\left|\xi_{1}\left(d_{1}-1\right) / C_{1}\right|^{1 / 2}$.

The vectors $c=\left(c^{A}\right)$ and $\bar{c}=\left(\bar{c}^{A}\right)$ satisfy the linear constraints

$$
U^{r}(c)=U^{r}(\bar{c})=0, \quad r=s, 1,
$$

(for $r=s$ see (3.38)) and the zero-energy constraint

$$
C_{1} \frac{d_{1}}{d_{1}-1}=2 E_{T}+h_{\alpha \beta} c^{\alpha} c^{\beta}+\sum_{i=2}^{n} d_{i}\left(c^{i}\right)^{2}+\frac{1}{d_{1}-1}\left(\sum_{i=2}^{n} d_{i} c^{i}\right)^{2} .
$$

\subsubsection{Special solutions for block-orthogonal set of vectors $U^{s}$}

Let us consider block-orthogonal case: (3.3), (3.4). In this case we get

$$
f_{s}=\left(\bar{f}_{s}\right)^{b_{s}}
$$

where $b_{s}=2 \sum_{s^{\prime} \in S} A^{s s^{\prime}},\left(A^{s s^{\prime}}\right)=\left(A_{s s^{\prime}}\right)^{-1}$ and

$$
\bar{f}_{s}(u)= \begin{cases}R_{s} \sinh \left(\sqrt{C_{s}}\left(u-u_{s}\right)\right), & C_{s}>0, \eta_{s} \varepsilon_{s}<0 \\ R_{s} \sin \left(\sqrt{\left|C_{s}\right|}\left(u-u_{s}\right)\right), & C_{s}<0, \eta_{s} \varepsilon_{s}<0 \\ R_{s} \cosh \left(\sqrt{C_{s}}\left(u-u_{s}\right)\right), & C_{s}>0, \eta_{s} \varepsilon_{s}>0 \\ \frac{\left|Q^{s}\right|}{\left|\nu_{s}\right|}\left(u-u_{s}\right), & C_{s}=0, \eta_{s} \varepsilon_{s}<0\end{cases}
$$

where $R_{s}=\left|Q_{s}\right| /\left(\left|\nu_{s}\right|\left|C_{s}\right|^{1 / 2}\right)$,

$$
\eta_{s} \nu_{s}^{2}=b_{s} h_{s}
$$

$\eta_{s}= \pm 1, C_{s}, u_{s}$ are constants, $s \in S$. The constants $C_{s}, u_{s}$ are coinciding inside the blocks: $u_{s}=u_{s^{\prime}}, C_{s}=C_{s^{\prime}}, s, s^{\prime} \in S_{i}, i=1, \ldots, k$. The ratios $\varepsilon_{s} Q_{s}^{2} /\left(b_{s} h_{s}\right)$ also coincide inside the blocks, or, equivalently,

$$
\frac{\varepsilon_{s} Q_{s}^{2}}{b_{s} h_{s}}=\frac{\varepsilon_{s^{\prime}} Q_{s^{\prime}}^{2}}{b_{s^{\prime}} h_{s^{\prime}}}, \quad s, s^{\prime} \in S_{i}, \quad i=1, \ldots, k .
$$


For energy integration constant (3.41) we get

$$
E_{T}=\frac{1}{2} \sum_{s \in S} C_{s} b_{s} h_{s}
$$

The solution (4.13)-(4.15) with a block-orthogonal set of $U^{s}$-vectors was obtained in [85, 86] (for non-composite case see also earlier paper by K.A. Bronnikov [84]). The generalized KM algebra corresponding to the generalized Cartan matrix $A$ in this case is semisimple. In the special orthogonal (or $A_{1} \oplus \cdots \oplus A_{1}$ ) case when: $\left|S_{1}\right|=\cdots=\left|S_{k}\right|=1$, the solution was obtained in [47].

Thus, here we presented a large class of exact solutions for invertible generalized Cartan matrices (e.g. corresponding to hyperbolic KM algebras). These solutions are governed by Toda-type equations. They are integrable in quadratures for finite-dimensional semisimple Lie algebras [87, 88, 89, 90, 91] in agreement with Adler-van Moerbeke criterion [91] (see also [92]).

The problem of integrability of Toda-chains related to Lorentzian (e.g. hyperbolic) KM algebras is much more complicated than in the Euclidean case. This is supported by the result from [93] (based on calculation of the Kovalevskaya exponents) where it was shown that the known cases of algebraic integrability for Euclidean Toda chains have no direct analogues in the case of spaces with pseudo-Euclidean metrics because the full-parameter expansions of the general solution contain complex powers of the independent variable. A similar result, using the Painlevé property, was obtained earlier for 2-dimensional Toda chains related to hyperbolic KM algebras [94].

Remark 4.1. It was shown in [95] that all supergravity billiards corresponding to sigma-models on any $U / H$ non compact-symmetric space and obtained by compactifying supergravity to $D=3$ are fully integrable. As far as we know this result could not be reformulated in terms of integrability of Toda-chains corresponding to certain Lorentzian (e.g. hyperbolic) KM algebras.

\subsection{Examples of $S$-brane solutions}

\section{Example 4.1. $S$-brane solution governed by $E_{10}$ Toda chain}

Let us consider the $B_{16}$-model in 16-dimensional pseudo-Euclidean space of signature $(-,+$, $\ldots,+)$ with six forms $F^{4}, \ldots, F^{9}$ and five scalar fields $\varphi^{1}, \ldots, \varphi^{5}$, see (3.21). Recall that for two branes corresponding to $F^{a}$ and $F^{b}$ forms the orthogonal (or $\left(A_{1}+A_{1}\right)$-) intersection rules read $[47,46]$ :

$$
\begin{aligned}
& (a-1)_{e} \cap_{o}(b-1)_{e}=N(a, b)=\min (a, b)-3, \\
& (a-1)_{e} \cap_{o}(D-b-1)_{m}=a-1-N(a, b),
\end{aligned}
$$

where $d_{v} \cap_{o} d_{v^{\prime}}^{\prime}$ denotes the dimension of orthogonal intersection for two branes with the dimensions of their worldvolumes being $d$ and $d^{\prime} . \quad d_{v} \cap_{o} d_{v^{\prime}}^{\prime}$ coincides with the symbol $\Delta\left(s, s^{\prime}\right)$ from $(3.13)^{1}$. The subscripts $v, v^{\prime}=e, m$ here indicate whether the brane is electric $(e)$ or magnetic $(m)$ one. In what follows we will be interested in the following orthogonal intersections: $4_{e} \cap_{o} 4_{e}=2,4_{e} \cap_{o} 5_{e}=2,4_{e} \cap_{o} 11_{m}=3,5_{e} \cap_{o} 11_{m}=4$.

Here we deal with $10\left(S\right.$-) branes: eight electric branes $s_{1}, s_{2}, s_{3}, s_{4}, s_{5}, s_{6}, s_{8}, s_{9}$ corresponding to 5 -form $F^{5}$, one electric brane $s_{7}$ corresponding to 6 -form $F^{6}$ and one magnetic brane $s_{10}$ corresponding to 4 -form $F^{4}$. The brane sets are as follows: $I_{1}=\{3,4,10,12\}, I_{2}=\{1,6,7,12\}$, $I_{3}=\{8,9,10,12\}, I_{4}=\{1,2,3,12\}, I_{5}=\{5,6,10,12\}, I_{6}=\{1,4,8,12\}, I_{7}=\{2,7,10,12,13\}$, $I_{8}=\{3,6,8,12\}, I_{9}=\{1,10,11,12\}, I_{10}=\{1,2,3,4,5,6,7,8,9,10,11\}$.

It may be verified that these sets do obey $E_{10}$ intersection rules following from the relations (3.14) (with $I_{s_{i}}=I_{i}$ ) and the Dynkin diagram from Fig. 1.

\footnotetext{
${ }^{1}$ Here as in [46] our notations differ from those adopted in string theory. For example for intersection of M2and M5-branes we write $3 \cap_{o} 6=2$ instead of $2 \cap 5=1$.
} 
123456789

Fig. 1. Dynkin diagram for $E_{10}$ hyperbolic KM algebra

Now we present a cosmological $S$-brane solution from Subsection 4.2.1 for the configuration of ten branes under consideration. In what follows the relations $\varepsilon_{s}=+1$ and $h_{s}=1 / 2, s \in S$, are used.

The metric (4.8) reads:

$$
\begin{aligned}
& g=\left[\left(\prod_{s \neq 7,10} f_{s}\right)^{4} f_{7}^{5} f_{10}^{11}\right]^{1 / 14}\left\{-e^{2 c^{0} t+2 \bar{c}^{0}} d t \otimes d t+\left(f_{2} f_{4} f_{6} f_{9} f_{10}\right)^{-1} e^{2 c^{1} t+2 \bar{c}^{1}} d x^{1} \otimes d x^{1}\right. \\
& +\left(f_{4} f_{7} f_{10}\right)^{-1} e^{2 c^{2} t+2 \bar{c}^{2}} d x^{2} \otimes d x^{2}+\left(f_{1} f_{4} f_{8} f_{10}\right)^{-1} e^{2 c^{3} t+2 \bar{c}^{3}} d x^{3} \otimes d x^{3} \\
& +\left(f_{1} f_{6} f_{10}\right)^{-1} e^{2 c^{4} t+2 \bar{c}^{4}} d x^{4} \otimes d x^{4}+\left(f_{5} f_{10}\right)^{-1} e^{2 c^{5} t+2 \bar{c}^{5}} d x^{5} \otimes d x^{5} \\
& +\left(f_{2} f_{5} f_{8} f_{10}\right)^{-1} e^{2 c^{6} t+2 \bar{c}^{6}} d x^{6} \otimes d x^{6}+\left(f_{2} f_{7} f_{10}\right)^{-1} e^{2 c^{7} t+2 \bar{c}^{7}} d x^{7} \otimes d x^{7} \\
& +\left(f_{3} f_{6} f_{8} f_{10}\right)^{-1} e^{2 c^{8} t+2 \bar{c}^{8}} d x^{8} \otimes d x^{8}+\left(f_{3} f_{10}\right)^{-1} e^{2 c^{9} t+2 \bar{c}^{9}} d x^{9} \otimes d x^{9} \\
& +\left(f_{1} f_{3} f_{5} f_{7} f_{9} f_{10}\right)^{-1} e^{2 c^{10} t+2 \bar{c}^{10}} d x^{10} \otimes d x^{10}+\left(f_{9} f_{10}\right)^{-1} e^{2 c^{11} t+2 \bar{c}^{11}} d x^{11} \otimes d x^{11} \\
& +\left(\prod_{s=1}^{9} f_{s}\right)^{-1} e^{2 c^{12} t+2 \bar{c}^{12}} d x^{12} \otimes d x^{12}+f_{7}^{-1} e^{2 c^{13} t+2 \bar{c}^{13}} d x^{13} \otimes d x^{13} \\
& \left.+e^{2 c^{14} t+2 \bar{c}^{14}} d x^{14} \otimes d x^{14}+e^{2 c^{15} t+2 \bar{c}^{15}} d x^{15} \otimes d x^{15}\right\} \text {. }
\end{aligned}
$$

For scalar fields (4.9) we get

$$
\varphi^{\alpha}=\frac{1}{2}\left[-\lambda_{5 \alpha}\left(\sum_{s \neq 7,10} \ln f_{s}\right)-\lambda_{6 \alpha} \ln f_{7}+\lambda_{4 \alpha} \ln f_{10}\right]+c_{\varphi}^{\alpha} t+\bar{c}_{\varphi}^{\alpha}, \quad \alpha=1, \ldots, 5 .
$$

Here we used the relations $\lambda_{a}^{\alpha}=-\lambda_{a \alpha}$.

The form fields (see (4.10) and (4.11)) are as follows

$$
\begin{aligned}
F^{4}= & Q_{10} d x^{12} \wedge d x^{13} \wedge d x^{14} \wedge d x^{15} \\
F^{5}= & Q_{1} f_{1}^{-2} f_{2} d t \wedge d x^{3} \wedge d x^{4} \wedge d x^{10} \wedge d x^{12}+Q_{2} f_{1} f_{2}^{-2} f_{3} d t \wedge d x^{1} \wedge d x^{6} \wedge d x^{7} \wedge d x^{12} \\
& +Q_{3} f_{2} f_{3}^{-2} f_{4} d t \wedge d x^{8} \wedge d x^{9} \wedge d x^{10} \wedge d x^{12}+Q_{4} f_{3} f_{4}^{-2} f_{5} d t \wedge d x^{1} \wedge d x^{2} \wedge d x^{3} \wedge d x^{12} \\
& +Q_{5} f_{4} f_{5}^{-2} f_{6} d t \wedge d x^{5} \wedge d x^{6} \wedge d x^{10} \wedge d x^{12}+Q_{6} f_{5} f_{6}^{-2} f_{7} d t \wedge d x^{1} \wedge d x^{4} \wedge d x^{8} \wedge d x^{12} \\
& +Q_{8} f_{7} f_{8}^{-2} f_{9} d t \wedge d x^{3} \wedge d x^{6} \wedge d x^{8} \wedge d x^{12}+Q_{9} f_{8} f_{9}^{-2} d t \wedge d x^{1} \wedge d x^{10} \wedge d x^{11} \wedge d x^{12} \\
F^{6}= & Q_{7} f_{6} f_{7}^{-2} f_{8} f_{10} d t \wedge d x^{2} \wedge d x^{7} \wedge d x^{10} \wedge d x^{12} \wedge d x^{13}
\end{aligned}
$$

where $Q_{s} \neq 0, s=1, \ldots, 10$. Here

$$
c^{0}=\sum_{j=1}^{15} c^{j}, \quad \bar{c}^{0}=\sum_{j=1}^{15} \bar{c}^{j}
$$

$f_{s}=\exp \left(-q^{s}(t)\right)$ and $q^{s}(t)$ obey Toda-type equations

$$
\ddot{q}^{s}=-2 Q_{s}^{2} \exp \left(\sum_{s^{\prime}=1}^{10} A_{s s^{\prime}} q^{s^{\prime}}\right), \quad s=1, \ldots, 10,
$$


where $\left(A_{s s^{\prime}}\right)$ is the Cartan matrix for the KM algebra $E_{10}$ (with the Dynkin diagram from Fig. 1) and the energy integration constant

$$
E_{T}=\frac{1}{8} \sum_{s, s^{\prime}=1}^{10} A_{s s^{\prime}} \dot{q^{s}} \dot{q^{s^{\prime}}}+\frac{1}{2} \sum_{s=1}^{10} Q_{s}^{2} \exp \left(\sum_{s^{\prime}=1}^{10} A_{s s^{\prime}} q^{s^{\prime}}\right),
$$

obeys the constraint

$$
2 E_{T}-\sum_{\alpha=1}^{5}\left(c_{\varphi}^{\alpha}\right)^{2}+\sum_{i=1}^{15}\left(c^{i}\right)^{2}-\left(\sum_{i=1}^{15} c^{i}\right)^{2}=0 .
$$

The brane constraints (3.38) are in our case

$$
\begin{array}{lll}
U^{1}(c)=c^{3}+c^{4}+c^{10}+c^{12}-\sum_{\alpha=1}^{5} \lambda_{5 \alpha} c_{\varphi}^{\alpha}=0, & U^{1}(\bar{c})=0, \\
U^{2}(c)=c^{1}+c^{6}+c^{7}+c^{12}-\sum_{\alpha=1}^{5} \lambda_{5 \alpha} c_{\varphi}^{\alpha}=0, & U^{2}(\bar{c})=0, \\
U^{3}(c)=c^{8}+c^{9}+c^{10}+c^{12}-\sum_{\alpha=1}^{5} \lambda_{5 \alpha} c_{\varphi}^{\alpha}=0, & U^{3}(\bar{c})=0, \\
U^{4}(c)=c^{1}+c^{2}+c^{3}+c^{12}-\sum_{\alpha=1}^{5} \lambda_{5 \alpha} c_{\varphi}^{\alpha}=0, & U^{4}(\bar{c})=0, \\
U^{5}(c)=c^{5}+c^{6}+c^{10}+c^{12}-\sum_{\alpha=1}^{5} \lambda_{5 \alpha} c_{\varphi}^{\alpha}=0, & U^{5}(\bar{c})=0, \\
U^{6}(c)=c^{1}+c^{4}+c^{8}+c^{12}-\sum_{\alpha=1}^{5} \lambda_{5 \alpha} c_{\varphi}^{\alpha}=0, & U^{6}(\bar{c})=0, \\
U^{7}(c)=c^{2}+c^{7}+c^{10}+c^{12}+c^{13}-\sum_{\alpha=1}^{5} \lambda_{6 \alpha} c_{\varphi}^{\alpha}=0, & U^{7}(\bar{c})=0, \\
U^{8}(c)=c^{3}+c^{6}+c^{8}+c^{12}-\sum_{\alpha=1}^{5} \lambda_{5 \alpha} c_{\varphi}^{\alpha}=0, & \\
U^{10}(c)=\sum_{i=1}^{11} c^{i}+\sum_{\alpha=1}^{5} \lambda_{4 \alpha} c_{\varphi}^{\alpha}=0, & U^{8}(\bar{c})=0, \\
U^{9}(c)=c^{1}+c^{10}+c^{11}+c^{12}-\sum_{5 \alpha} c_{\varphi}^{\alpha}=0, & U^{9}(\bar{c})=0, \\
\end{array}
$$

Remark 4.2. For a special choice of integration constants $c^{i}=0$ and $c_{\varphi}^{\alpha}=0$, we get a solution governed by $E_{10}$ Toda chain with the energy constraint $E_{T}=0$. According to the result from [23] we obtain a never ending asymptotical oscillating behavior of scale factors which is described by the motion of a point-like particle in a billiard $B \subset H^{9}$. This billiard has a finite volume since $E_{10}$ is hyperbolic.

Special 1-block solution. Now we consider a special 1-block solution (see Subsection 4.2.3). This solution is valid when a special set of charges is considered (see (4.16)):

$$
Q_{s}^{2}=Q^{2}\left|b_{s}\right|
$$


where $Q \neq 0$ and $[38]$

$$
b_{s}=2 \sum_{s^{\prime}=1}^{10} A^{s s^{\prime}}=-60,-122,-186,-252,-320,-390,-462,-306,-152,-230,
$$

$s=1, \ldots, 10$. Recall that $\left(A^{s s^{\prime}}\right)=\left(A_{s s^{\prime}}\right)^{-1}$.

In this case $f_{s}=(\bar{f})^{b_{s}}$, where

$$
\bar{f}(t)= \begin{cases}|Q| \sqrt{2 / C} \sinh \left(\sqrt{C}\left(t-t_{0}\right)\right), & C>0 \\ |Q| \sqrt{2 /|C|} \sin \left(\sqrt{|C|}\left(t-t_{0}\right)\right), & C<0 \\ |Q| \sqrt{2}\left(t-t_{0}\right), & C=0\end{cases}
$$

and $t_{0}$ is a constant.

From (4.17) we get

$$
E_{T}=-620 C
$$

where relation $\sum_{s=1}^{10} b_{s}=-2480$ was used.

For the special solution under consideration the electric monomials in (4.18) have a simpler form

$$
\mathcal{F}^{s}=Q_{s} \bar{f}^{-2} d t \wedge \tau\left(I_{s}\right)
$$

where $s=1,2, \ldots, 9$.

Solution with one harmonic function. Let $C=0$ and all $c^{i}=\bar{c}^{i}=0, c_{\varphi}^{\alpha}=\bar{c}_{\varphi}^{\alpha}=0$. In this case $H=\bar{f}(t)=|Q| \sqrt{2}\left(t-t_{0}\right)>0$ is a harmonic function on the 1-dimensional manifold $\left(\left(t_{0},+\infty\right),-d t \otimes d t\right)$ and our solution coincides with the 1-block solution (3.6)-(3.10) (if $\operatorname{sign} \nu_{s}=$ $-\operatorname{sign} Q_{s}$ for all $\left.s\right)$.

Example 4.2. $S$-brane solution governed by $\boldsymbol{H} \boldsymbol{A}_{2}^{(1)}$ Toda chain. Now we consider the $B_{11}$-model in 11-dimensional pseudo-Euclidean space of signature $(-,+, \ldots,+)$ with 4 -form $F^{4}$.

Here we deal with four electric branes ( $S M 2$-branes) $s_{1}, s_{2}, s_{3}, s_{4}$ corresponding to the 4form $F^{4}$. The brane sets are the following ones: $I_{1}=\{1,2,3\}, I_{2}=\{4,5,6\}, I_{3}=\{7,8,9\}$, $I_{4}=\{1,4,10\}$.

It may be verified that these sets obey the intersection rules corresponding to the hyperbolic KM algebra $H A_{2}^{(1)}$ with the following Cartan matrix

$$
A=\left(\begin{array}{cccc}
2 & -1 & -1 & 0 \\
-1 & 2 & -1 & 0 \\
-1 & -1 & 2 & -1 \\
0 & 0 & -1 & 2
\end{array}\right)
$$

(see (3.14) with $\left.I_{s_{i}}=I_{i}\right)$.

Now we give a cosmological $S$-brane solution from Subsection 4.2.1 for the configuration of four branes under consideration. In what follows the relations $\varepsilon_{s}=+1$ and $h_{s}=1 / 2, s \in S$, are used.

The metric (4.8) reads:

$$
\begin{aligned}
g= & \left(f_{1} f_{2} f_{3} f_{4}\right)^{1 / 3}\left\{-e^{2 c^{0} t+2 \bar{c}^{0}} d t \otimes d t+\left(f_{1} f_{4}\right)^{-1} e^{2 c^{1} t+2 \bar{c}^{1}} d x^{1} \otimes d x^{1}+f_{1}^{-1} e^{2 c^{2} t+2 \bar{c}^{2}} d x^{2} \otimes d x^{2}\right. \\
& +f_{1}^{-1} e^{2 c^{3} t+2 \bar{c}^{3}} d x^{3} \otimes d x^{3}+\left(f_{2} f_{4}\right)^{-1} e^{2 c^{4} t+2 \bar{c}^{4}} d x^{4} \otimes d x^{4}+f_{2}^{-1} e^{2 c^{5} t+2 \bar{c}^{5}} d x^{5} \otimes d x^{5}
\end{aligned}
$$




$$
\begin{aligned}
& +f_{2}^{-1} e^{2 c^{6} t+2 \bar{c}^{6}} d x^{6} \otimes d x^{6}+f_{3}^{-1} e^{2 c^{7} t+2 \bar{c}^{7}} d x^{7} \otimes d x^{7}+f_{3}^{-1} e^{2 c^{8} t+2 \bar{c}^{8}} d x^{8} \otimes d x^{8} \\
& \left.+f_{3}^{-1} e^{2 c^{9} t+2 \bar{c}^{9}} d x^{9} \otimes d x^{9}+f_{4}^{-1} e^{2 c^{10} t+2 \bar{c}^{10}} d x^{10} \otimes d x^{10}\right\}
\end{aligned}
$$

The form field (see (4.10)) is as follows

$$
\begin{aligned}
F^{4}= & Q_{1} f_{1}^{-2} f_{2} f_{3} d t \wedge d x^{1} \wedge d x^{2} \wedge d x^{3}+Q_{2} f_{1} f_{2}^{-2} f_{3} d t \wedge d x^{4} \wedge d x^{5} \wedge d x^{6} \\
& +Q_{3} f_{1} f_{2} f_{3}^{-2} d t \wedge d x^{7} \wedge d x^{8} \wedge d x^{9}+Q_{4} f_{3} f_{4}^{-2} d t \wedge d x^{1} \wedge d x^{4} \wedge d x^{10}
\end{aligned}
$$

where $Q_{s} \neq 0, s=1, \ldots, 4$. Here

$$
c^{0}=\sum_{j=1}^{10} c^{j}, \quad \bar{c}^{0}=\sum_{j=1}^{10} \bar{c}^{j},
$$

$f_{s}=\exp \left(-q^{s}(t)\right)$ and $q^{s}(t)$ obey the Toda-type equations

$$
\ddot{q^{s}}=-2 Q_{s}^{2} \exp \left(\sum_{s^{\prime}=1}^{4} A_{s s^{\prime}} q^{s^{\prime}}\right)
$$

$s=1, \ldots, 4$, where $\left(A_{s s^{\prime}}\right)$ is the Cartan matrix (4.19) for the $\mathrm{KM}$ algebra $H A_{2}^{(1)}$ and the energy integration constant

$$
E_{T}=\frac{1}{8} \sum_{s, s^{\prime}=1}^{4} A_{s s^{\prime}} \dot{q} q^{\dot{s}^{\prime}}+\frac{1}{2} \sum_{s=1}^{4} Q_{s}^{2} \exp \left(\sum_{s^{\prime}=1}^{4} A_{s s^{\prime}} q^{s^{\prime}}\right),
$$

obeys the constraint

$$
2 E_{T}+\sum_{i=1}^{10}\left(c^{i}\right)^{2}-\left(\sum_{i=1}^{10} c^{i}\right)^{2}=0 .
$$

The brane constraints (3.38) read in this case as follows

$$
\begin{array}{ll}
U^{1}(c)=c^{1}+c^{2}+c^{3}=0, & U^{1}(\bar{c})=0, \\
U^{2}(c)=c^{4}+c^{5}+c^{6}=0, & U^{2}(\bar{c})=0, \\
U^{3}(c)=c^{7}+c^{8}+c^{9}=0, & U^{3}(\bar{c})=0, \\
U^{4}(c)=c^{1}+c^{4}+c^{10}=0, & U^{4}(\bar{c})=0 .
\end{array}
$$

Since $F^{4} \wedge F^{4}=0$ this solution also obeys equations of motion of 11-dimensional supergravity.

Special 1-block solution. Now we consider a special 1-block solution (see subsection 4.2.3). This solution is valid when a special set of charges is considered (see (4.16)):

$$
Q_{s}^{2}=Q^{2}\left|b_{s}\right|
$$

where $Q \neq 0$ and

$$
b_{s}=2 \sum_{s^{\prime}=1}^{4} A^{s s^{\prime}}=-12,-12,-14,-6 .
$$

In this case $f_{s}=(\bar{f})^{b_{s}}$, where $\bar{f}$ is the same as in (4.1).

For the energy integration constant we have

$$
E_{T}=-11 C,
$$

(see (4.17)). 
Example 4.3. $S$-brane solution governed by $\boldsymbol{P}_{10}$ Toda chain with $\boldsymbol{E}_{\boldsymbol{T}}=\mathbf{0}$. Now we consider the $B_{11}$-model in 11-dimensional pseudo-Euclidean space of signature $(-,+, \ldots,+)$ with 4 -form $F^{4}$.

Here we deal with ten electric branes ( $S M 2$-branes) $s_{1}, \ldots, s_{10}$ corresponding to the 4 form $F^{4}$. The brane sets are taken from $[96,6]$ as: $I_{1}=\{1,4,7\}, I_{2}=\{8,9,10\}, I_{3}=\{2,5,7\}$, $I_{4}=\{4,6,10\}, I_{5}=\{2,3,9\}, I_{6}=\{1,2,8\}, I_{7}=\{1,3,10\}, I_{8}=\{4,5,8\}, I_{9}=\{3,6,7\}$, $I_{10}=\{5,6,9\}$.

These sets obey the intersection rules corresponding to the Lorentzian KM algebra $P_{10}$ (we call it Petersen algebra) with the following Cartan matrix

$$
A=\left(\begin{array}{cccccccccc}
2 & -1 & 0 & 0 & -1 & 0 & 0 & 0 & 0 & -1 \\
-1 & 2 & -1 & 0 & 0 & 0 & 0 & 0 & -1 & 0 \\
0 & -1 & 2 & -1 & 0 & 0 & -1 & 0 & 0 & 0 \\
0 & 0 & -1 & 2 & -1 & -1 & 0 & 0 & 0 & 0 \\
-1 & 0 & 0 & -1 & 2 & 0 & 0 & -1 & 0 & 0 \\
0 & 0 & 0 & -1 & 0 & 2 & 0 & 0 & -1 & -1 \\
0 & 0 & -1 & 0 & 0 & 0 & 2 & -1 & 0 & -1 \\
0 & 0 & 0 & 0 & -1 & 0 & -1 & 2 & -1 & 0 \\
0 & -1 & 0 & 0 & 0 & -1 & 0 & -1 & 2 & 0 \\
-1 & 0 & 0 & 0 & 0 & -1 & -1 & 0 & 0 & 2
\end{array}\right) .
$$

The Dynkin diagram for this Cartan matrix could be represented by the Petersen graph ("a star inside a pentagon"). $P_{10}$ is the Lorentzian KM algebra. It is a subalgebra of $E_{10}[96,6]$.

Let us present an $S$-brane solution for the configuration of 10 electric branes under consideration. The metric (4.8) reads:

$$
\begin{aligned}
g= & \left(\prod_{s=1}^{10} f_{s}\right)^{1 / 3}\left\{-d t \otimes d t+\left(f_{1} f_{6} f_{7}\right)^{-1} d x^{1} \otimes d x^{1}+\left(f_{3} f_{5} f_{6}\right)^{-1} d x^{2} \otimes d x^{2}\right. \\
& +\left(f_{5} f_{7} f_{9}\right)^{-1} d x^{3} \otimes d x^{3}+\left(f_{1} f_{4} f_{8}\right)^{-1} d x^{4} \otimes d x^{4}+\left(f_{3} f_{8} f_{10}\right)^{-1} d x^{5} \otimes d x^{5} \\
& +\left(f_{4} f_{9} f_{10}\right)^{-1} d x^{6} \otimes d x^{6}+\left(f_{1} f_{3} f_{9}\right)^{-1} d x^{7} \otimes d x^{7}+\left(f_{2} f_{6} f_{8}\right)^{-1} d x^{8} \otimes d x^{8} \\
& \left.+\left(f_{2} f_{5} f_{10}\right)^{-1} d x^{9} \otimes d x^{9}+\left(f_{2} f_{4} f_{7}\right)^{-1} d x^{10} \otimes d x^{10}\right\} .
\end{aligned}
$$

The form field (see (4.10)) is the following

$$
\begin{aligned}
F^{4}= & Q_{1} f_{1}^{-2} f_{2} f_{5} f_{10} d t \wedge d x^{1} \wedge d x^{4} \wedge d x^{7}+Q_{2} f_{1} f_{2}^{-2} f_{3} f_{9} d t \wedge d x^{8} \wedge d x^{9} \wedge d x^{10} \\
& +Q_{3} f_{2} f_{3}^{-2} f_{4} f_{7} d t \wedge d x^{2} \wedge d x^{5} \wedge d x^{7}+Q_{4} f_{3} f_{4}^{-2} f_{5} f_{6} d t \wedge d x^{4} \wedge d x^{6} \wedge d x^{10} \\
& +Q_{5} f_{1} f_{4} f_{5}^{-2} f_{8} d t \wedge d x^{2} \wedge d x^{3} \wedge d x^{9}+Q_{6} f_{4} f_{6}^{-2} f_{9} f_{10} d t \wedge d x^{1} \wedge d x^{2} \wedge d x^{8} \\
& +Q_{7} f_{3} f_{7}^{-2} f_{8} f_{10} d t \wedge d x^{1} \wedge d x^{3} \wedge d x^{10}+Q_{8} f_{5} f_{7} f_{8}^{-2} f_{9} d t \wedge d x^{4} \wedge d x^{5} \wedge d x^{8} \\
& +Q_{9} f_{2} f_{6} f_{8} f_{9}^{-2} d t \wedge d x^{3} \wedge d x^{6} \wedge d x^{7}+Q_{10} f_{1} f_{6} f_{7} f_{10}^{-2} d t \wedge d x^{5} \wedge d x^{6} \wedge d x^{9}
\end{aligned}
$$

where $Q_{s} \neq 0, s=1, \ldots, 10$. Here $f_{s}=\exp \left(-q^{s}(t)\right)$ and $q^{s}(t)$ obey the Toda-type equations

$$
\ddot{q}^{s}=-2 Q_{s}^{2} \exp \left(\sum_{s^{\prime}=1}^{10} A_{s s^{\prime}} q^{s^{\prime}}\right)
$$

where $\left(A_{s s^{\prime}}\right)$ is the Cartan matrix (4.20) for the KM algebra $P_{10}$ and the energy constraint

$$
E_{T}=\frac{1}{8} \sum_{s, s^{\prime}=1}^{10} A_{s s^{\prime}} \dot{q}^{s} \dot{q}^{s^{\prime}}+\frac{1}{2} \sum_{s=1}^{10} Q_{s}^{2} \exp \left(\sum_{s^{\prime}=1}^{10} A_{s s^{\prime}} q^{s^{\prime}}\right)=0
$$


is obeyed. Here we used the fact that the two sets of linear equations $U^{s}(c)=0, U^{s}(\bar{c})=0$, $s=1, \ldots, 10$, have trivial solutions: $c=0, \bar{c}=0$, due to the linear independence of vectors $U^{s}$.

Since $F^{4} \wedge F^{4}=0$, this solution also obeys the equations of motion of 11-dimensional supergravity.

Remark 4.3. As pointed out in [96] we do not obtain a never ending asymptotic oscillating behavior of the scale factors in this case since the Lorentzian KM algebra $P_{10}$ is not hyperbolic and the corresponding billiard $B \subset H^{9}$ has an infinite volume.

Special 1-block solution. Now we consider a special 1-block solution. The calculations give us the following relations

$$
b_{s}=2 \sum_{s^{\prime}=1}^{10} A^{s s^{\prime}}=-2, \quad s=1, \ldots, 10,
$$

and hence the special solution is valid (see (4.16)), when all charges are equal

$$
Q_{s}^{2}=Q^{2}
$$

where $Q \neq 0$. In this case all $f_{s}=\bar{f}^{-2}$, where

$$
\bar{f}(t)=|Q|\left(t-t_{0}\right),
$$

and $t_{0}$ is constant. The metric (4.3) may be rewritten using the synchronous time variable $t_{s}$ :

$$
g=-d t_{s} \otimes d t_{s}+A t_{s}^{2 / 7} \sum_{i=1}^{10} d x^{i} \otimes d x^{i},
$$

where $A>0$ and $t_{s}>0$. This metric coincides with the power-law, inflationary solution in the model with a one-component perfect fluid when the following equation of state is adopted: $p=\frac{2}{5} \rho$, where $p$ is pressure and $\rho$ is the density of fluid [97, 98].

\section{$5 \quad$ Black brane solutions}

In this section we consider the spherically symmetric case of the metric (4.13), i.e. we put $w=1$, $M_{1}=S^{d_{1}}, g^{1}=d \Omega_{d_{1}}^{2}$, where $d \Omega_{d_{1}}^{2}$ is the canonical metric on a unit sphere $S^{d_{1}}, d_{1} \geq 2$. In this case $\xi^{1}=d_{1}-1$. We put $M_{2}=\mathbb{R}, g^{2}=-d t \otimes d t$, i.e. $M_{2}$ is a time manifold.

Let $C_{1} \geq 0$. We consider solutions defined on some interval $\left[u_{0},+\infty\right)$ with a horizon at $u=+\infty$.

When the matrix $\left(h_{\alpha \beta}\right)$ is positive definite and

$$
2 \in I_{s}, \quad \forall s \in S,
$$

i.e. all branes have a common time direction $t$, the horizon condition singles out the unique solution with $C_{1}>0$ and linear asymptotics at infinity

$$
q^{s}=-\beta^{s} u+\bar{\beta}^{s}+o(1)
$$

$u \rightarrow+\infty$, where $\beta^{s}, \bar{\beta}^{s}$ are constants, $s \in S[42,43]$.

In this case

$$
c^{A} / \bar{\mu}=-\delta_{2}^{A}+h_{1} U^{1 A}+\sum_{s \in S} h_{s} b_{s} U^{s A}, \quad \beta^{s} / \bar{\mu}=2 \sum_{s^{\prime} \in S} A^{s s^{\prime}} \equiv b_{s},
$$


where $s \in S, A=(i, \alpha), \bar{\mu}=\sqrt{C_{1}}$, the matrix $\left(A^{s s^{\prime}}\right)$ is inverse of the generalized Cartan matrix $\left(A_{s s^{\prime}}\right)$ and $h_{1}=\left(U^{1}, U^{1}\right)^{-1}=d_{1} /\left(1-d_{1}\right)$.

Let us introduce a new radial variable $R=R(u)$ through the relations

$$
\exp (-2 \bar{\mu} u)=1-\frac{2 \mu}{R^{\bar{d}}}, \quad \mu=\bar{\mu} / \bar{d}>0
$$

where $u>0, R^{\bar{d}}>2 \mu, \bar{d}=d_{1}-1$. We put $\bar{c}^{A}=0$ and $q^{s}(0)=0, A=(i, \alpha), s \in S$. These relations guarantee the asymptotic flatness (for $R \rightarrow+\infty)$ of the $\left(2+d_{1}\right)$-dimensional section of the metric.

Let us denote $H_{s}=f_{s} e^{-\beta^{s} u}, s \in S$. Then, solutions (4.13)-(4.15) may be written as follows $[41,42,43]$

$$
\begin{aligned}
& g=\left(\prod_{s \in S} H_{s}^{2 h_{s} d\left(I_{s}\right) /(D-2)}\right)\left\{\left(1-\frac{2 \mu}{R^{\bar{d}}}\right)^{-1} d R \otimes d R+R^{2} d \Omega_{d_{1}}^{2}\right. \\
& \left.\quad-\left(\prod_{s \in S} H_{s}^{-2 h_{s}}\right)\left(1-\frac{2 \mu}{R^{\bar{d}}}\right) d t \otimes d t+\sum_{i=3}^{n}\left(\prod_{s \in S} H_{s}^{-2 h_{s} \delta_{i I_{s}}}\right) \hat{g}^{i}\right\}, \\
& \exp \left(\varphi^{\alpha}\right)=\prod_{s \in S} H_{s}^{h_{s} \chi_{s} \lambda_{a_{s}}^{\alpha}}
\end{aligned}
$$

where $F^{a}=\sum_{s \in S} \delta_{a_{s}}^{a} \mathcal{F}^{s}$, and

$$
\begin{aligned}
\mathcal{F}^{s} & =-\frac{Q_{s}}{R^{d_{1}}}\left(\prod_{s^{\prime} \in S} H_{s^{\prime}}^{-A_{s s^{\prime}}}\right) d R \wedge \tau\left(I_{s}\right), \quad s \in S_{e}, \\
\mathcal{F}^{s} & =Q_{s} \tau\left(\bar{I}_{s}\right), \quad s \in S_{m} .
\end{aligned}
$$

Here $Q_{s} \neq 0, h_{s}=K_{s}^{-1}, s \in S$, and the generalized Cartan matrix $\left(A_{s s^{\prime}}\right)$ is non-degenerate.

Functions $H_{s}>0$ obey the equations

$$
\begin{aligned}
& \frac{d}{d z}\left(\frac{(1-2 \mu z)}{H_{s}} \frac{d}{d z} H_{s}\right)=\bar{B}_{s} \prod_{s^{\prime} \in S} H_{s^{\prime}}^{-A_{s s^{\prime}},} \\
& H_{s}\left((2 \mu)^{-1}-0\right)=H_{s 0} \in(0,+\infty), \\
& H_{s}(+0)=1, \quad s \in S,
\end{aligned}
$$

where $H_{s}(z)>0, \mu>0, z=R^{-\bar{d}} \in\left(0,(2 \mu)^{-1}\right)$ and $\bar{B}_{s}=\varepsilon_{s} K_{s} Q_{s}^{2} / \bar{d}^{2} \neq 0$.

There exist solutions to equations (5.6)-(5.7) of polynomial type. The simplest example occurs in orthogonal case [58, 47] (for $d_{i}=1$ see also $\left.[56,57]\right):\left(U^{s}, U^{s^{\prime}}\right)=0$, for $s \neq s^{\prime}$, $s, s^{\prime} \in S$. In this case $\left(A_{s s^{\prime}}\right)=\operatorname{diag}(2, \ldots, 2)$ is a Cartan matrix for the semisimple Lie algebra $\mathbf{A}_{\mathbf{1}} \oplus \cdots \oplus \mathbf{A}_{\mathbf{1}}$ and

$$
H_{s}(z)=1+P_{s} z
$$

with $P_{s} \neq 0$, satisfying

$$
P_{s}\left(P_{s}+2 \mu\right)=-\bar{B}_{s}, \quad s \in S .
$$

(For earlier supergravity solutions see [99, 100] and references therein).

In $[84,86,101]$ this solution was generalized to a block-orthogonal case $(3.3),(3.4)$. In this case (5.9) is modified as follows

$$
H_{s}(z)=\left(1+P_{s} z\right)^{b_{s}},
$$


where $b_{s}$ are defined in (5.1) and parameters $P_{s}$ coincide inside blocks, i.e. $P_{s}=P_{s^{\prime}}$ for $s, s^{\prime} \in S_{i}$, $i=1, \ldots, k$. The parameters $P_{s} \neq 0$ satisfy the relations $[86,101,46]$

$$
P_{s}\left(P_{s}+2 \mu\right)=-\bar{B}_{s} / b_{s}, \quad s \in S,
$$

and the parameters $\bar{B}_{s} / b_{s}$ coincide inside blocks, i.e. $\bar{B}_{s} / b_{s}=\bar{B}_{s^{\prime}} / b_{s^{\prime}}$ for $s, s^{\prime} \in S_{i}, i=1, \ldots, k$.

Finite-dimensional Lie algebras. Let $\left(A_{s s^{\prime}}\right)$ be a Cartan matrix for a finite-dimensional semisimple Lie algebra $\mathcal{G}$. In this case all powers $b_{s}$ defined in (5.1) are natural numbers which coincide with the components of twice the dual Weyl vector in the basis of simple co-roots [4] and hence, all functions $H_{s}$ are polynomials, $s \in S$.

Conjecture 5.1. Let $\left(A_{s s^{\prime}}\right)$ be a Cartan matrix for a semisimple finite-dimensional Lie algebra $\mathcal{G}$. Then the solutions to equations (5.6)-(5.8) (if exist) have a polynomial structure:

$$
H_{s}(z)=1+\sum_{k=1}^{n_{s}} P_{s}^{(k)} z^{k},
$$

where $P_{s}^{(k)}$ are constants, $k=1, \ldots, n_{s} ; n_{s}=b_{s}=2 \sum_{s^{\prime} \in S} A^{s s^{\prime}} \in \mathbb{N}$ and $P_{s}^{\left(n_{s}\right)} \neq 0, s \in S$.

In the extremal case $(\mu=+0)$ an analogue of this conjecture was suggested previously in [76]. Conjecture 5.1 was verified for the $\mathbf{A}_{\mathbf{m}}$ and $\mathbf{C}_{\mathbf{m}+\mathbf{1}}$ Lie algebras in [42, 43]. Explicit expressions for polynomials corresponding to Lie algebras $C_{2}$ and $A_{3}$ were obtained in [102] and [103] respectively.

Hyperbolic KM algebras. Let $\left(A_{s s^{\prime}}\right)$ be a Cartan matrix for an infinite-dimensional hyperbolic KM algebra $\mathcal{G}$. In this case all powers in (5.1) are negative numbers and hence, we have no chance to get a polynomial structure for $H_{s}$. Here we are led to an open problem of seeking solutions to the set of "master" equations (5.6) with boundary conditions (5.7) and (5.8). These solutions define special solutions to Toda-chain equations corresponding to the hyperbolic KM algebra $\mathcal{G}$.

Example 5.1. Black hole solutions for $A_{1} \oplus A_{1}, A_{2}$ and $H_{2}(q, q) \mathrm{KM}$ algebras. Let us consider the 4-dimensional model governed by the action

$$
S=\int_{M} d^{4} z \sqrt{|g|}\left\{R[g]-\varepsilon g^{M N} \partial_{M} \varphi \partial_{N} \varphi-\frac{1}{2} e^{2 \lambda \varphi}\left(F^{1}\right)^{2}-\frac{1}{2} e^{-2 \lambda \varphi}\left(F^{2}\right)^{2}\right\} .
$$

Here $F^{1}$ and $F^{2}$ are 2-forms, $\varphi$ is scalar field and $\varepsilon= \pm 1$.

We consider a black brane solution defined on $\mathbb{R}_{*} \times S^{2} \times \mathbb{R}$ with two electric branes $s_{1}$ and $s_{2}$ corresponding to forms $F^{1}$ and $F^{2}$, respectively, with the sets $I_{1}=I_{2}=\{2\}$. Here $\mathbb{R}_{*}$ is subset of $\mathbb{R}, M_{1}=S^{2}, g^{1}=d \Omega_{2}^{2}$, is the canonical metric on $S^{2}, M_{2}=\mathbb{R}, g^{2}=-d t \otimes d t$ and $\varepsilon_{1}=\varepsilon_{2}=-1$.

The scalar products of $U$-vectors are (we identify $U^{i}=U^{s_{i}}$ ):

$$
\left(U^{1}, U^{1}\right)=\left(U^{2}, U^{2}\right)=\frac{1}{2}+\varepsilon \lambda^{2} \neq 0, \quad\left(U^{1}, U^{2}\right)=\frac{1}{2}-\varepsilon \lambda^{2} .
$$

The matrix $A$ from (3.11) is a generalized non-degenerate Cartan matrix if and only if

$$
\frac{2\left(U^{1}, U^{2}\right)}{\left(U^{2}, U^{2}\right)}=-q,
$$

or, equivalently,

$$
\varepsilon \lambda^{2}=\frac{2+q}{2(2-q)},
$$


where $q=0,1,3,4, \ldots$ This takes place when

$$
\varepsilon=+1, \quad q=0,1, \quad \varepsilon=-1, \quad q=3,4,5, \ldots
$$

and

$$
\lambda^{2}=\frac{2+q}{2|2-q|} .
$$

The first branch $(\varepsilon=+1)$ corresponds to finite dimensional Lie algebras $A_{1} \oplus A_{1}(q=0)$, $A_{2}(q=1)$ and the second one $(\varepsilon=-1)$ corresponds to hyperbolic KM algebras $H_{2}(q, q)$, $q=3,4, \ldots$. In the hyperbolic case the scalar field $\varphi$ is a phantom (ghost).

The black brane solution reads (see (5.2)-(5.4))

$$
\begin{aligned}
& g=\left(H_{1} H_{2}\right)^{h}\left\{\left(1-\frac{2 \mu}{R}\right)^{-1} d R \otimes d R+R^{2} d \Omega_{2}^{2}-\left(H_{1} H_{2}\right)^{-2 h}\left(1-\frac{2 \mu}{R}\right) d t \otimes d t\right\}, \\
& \exp (\varphi)=\left(H_{1} / H_{2}\right)^{\varepsilon \lambda h}, \\
& F^{s}=\frac{Q_{s}}{R^{2}} H_{s}^{-2}\left(H_{\bar{s}}\right)^{q} d t \wedge d R, \quad s=1,2 .
\end{aligned}
$$

Here $h=(2-q) / 2$ and $\bar{s}=2,1$ for $s=1,2$ respectively.

The moduli functions $H_{s}>0$ obey the equations (see (5.6))

$$
\frac{d}{d z}\left(\frac{(1-2 \mu z)}{H_{s}} \frac{d}{d z} H_{s}\right)=\frac{2 Q_{s}^{2}}{q-2} H_{s}^{-2}\left(H_{\bar{s}}\right)^{q}
$$

with the boundary conditions $H_{s}\left((2 \mu)^{-1}-0\right)=H_{s 0} \in(0,+\infty), H_{s}(+0)=1, s=1,2$, imposed. Here $\mu>0, z=1 / R \in\left(0,(2 \mu)^{-1}\right)$. For $q=0,1$ the solutions to equations (5.15) with the boundary conditions imposed were given in [41, 42, 43]. They are polynomials of degrees 1 and 2 for $q=0$ and $q=1$, respectively. For $q=3,4, \ldots$ the exact solutions to equations (5.15) are not known yet.

Special solution with $Q_{1}^{2}=Q_{2}^{2}$. Now we consider the special one-block solution with the functions $H_{s}$ obeying (5.10) and (5.11). Since $b_{s}=2 /(2-q)$ and $\bar{B}_{s}=2 Q_{s}^{2} /(q-2)$ it takes place when $Q_{1}^{2}=Q_{2}^{2}=Q^{2}>0$. The moduli functions read

$$
H_{s}=H^{2 /(2-q)}, \quad H=1+P z,
$$

where $z=1 / R$ and $q \neq 2$. These functions obey $H_{s}(z)>0$ for $z \in\left[0,(2 \mu)^{-1}\right]$ if $P>-2 \mu$ $(\mu>0)$. Due to this inequality and the relation $P(P+2 \mu)=Q^{2}$ (following from (5.11) ) we get

$$
P=-\mu+\sqrt{\mu^{2}+Q^{2}}>0 .
$$

In this special case the solution (5.12)-(5.14) has the following form:

$$
\begin{aligned}
& g=H^{2}\left\{\left(1-\frac{2 \mu}{R}\right)^{-1} d R \otimes d R+R^{2} d \Omega_{2}^{2}-H^{-4}\left(1-\frac{2 \mu}{R}\right) d t \otimes d t\right\}, \\
& \varphi=0 \\
& F^{s}=\frac{Q_{s}}{H^{2} R^{2}} d t \wedge d R, \quad s=1,2 .
\end{aligned}
$$

Remarkably, this special solution does not depend upon $q$. The metric (5.16) coincides with the metric of the Reissner-Nordström solution (when the Maxwell 2-form is $F=\sqrt{2} Q(H R)^{-2} d t \wedge$ $d R)$. 
In the extremal case $\mu \rightarrow+0$ we are lead to the special case of a Majumdar-Papapetrou type solution

$$
\begin{aligned}
& g=H^{2} \hat{g}^{0}-H^{-2} d t \otimes d t, \\
& \varphi=0, \\
& F^{s}=\nu_{s} d H^{-1} \wedge d t
\end{aligned}
$$

where $g^{0}=\sum_{i=1}^{3} d x^{i} \otimes d x^{i}, H$ is a harmonic function on $M_{0}=\mathbb{R}^{3}$ and $\nu_{s}^{2}=1, s=1,2$. Here $\nu_{s}=-Q_{s} / Q$.

\section{Conclusions}

Here we reviewed several families of exact solutions in multidimensional gravity with a set of scalar fields and fields of forms related to non-singular (e.g. hyperbolic) KM algebras.

The solutions describe composite electromagnetic branes defined on warped products of Ricciflat, or sometimes Einstein, spaces of arbitrary dimensions and signatures. The metrics are block-diagonal and all scale factors, scalar fields and fields of forms depend on points of some manifold $M_{0}$. The solutions include those depending upon harmonic functions, S-branes and spherically-symmetric solutions (e.g. black-branes). Our approach is based on the sigma-model representation obtained in [48] under the rather general assumption on intersections of composite branes (when stress-energy tensor has a diagonal structure).

We were dealing with rather general intersection rules [47] governed by invertible generalized Cartan matrix corresponding to the certain generalized KM Lie algebra $\mathcal{G}$. For $\mathcal{G}=\mathbf{A}_{\mathbf{1}} \oplus \cdots \oplus \mathbf{A}_{\mathbf{1}}$ ( $r$ terms) we get the well-known standard (e.g. supersymmetry preserving) intersection rules $[53,54,55,48]$.

We have also considered a class of special "block-orthogonal" solutions corresponding to semisimple KM algebras and governed by several harmonic functions. Certain examples of 1-block solutions (e.g. corresponding to $\mathrm{KM}$ algebras $\left.H_{2}(q, q), A E_{3}\right)$ were considered.

In the one-block case a generalization of the solutions to those governed by several functions of one harmonic function $H$ and obeying Toda-type equations was presented.

For finite-dimensional (semi-simple) Lie algebras we are led to integrable Lagrange systems while the Toda chains corresponding to infinite-dimensional (non-singular) KM algebras are not well studied yet. Some examples of $S$-brane solutions corresponding to Lorentzian KM algebras $H A_{2}^{(1)}=A_{2}^{++}, E_{10}$ and $P_{10}$ were presented.

We have also considered general classes of cosmological-type solutions (e.g. $S$-brane and spherically symmetric solutions) governed by Toda-type equations, containing black brane configurations as a special case. The "master" equations for moduli functions have polynomial solutions in the finite-dimensional case (according to our conjecture [41, 42, 43]), while in the infinite-dimensional case we have only a special family of the so-called block-orthogonal solutions corresponding to semi-simple non-singular KM algebras. Examples of 4-dimensional dilatonic black hole solutions corresponding to KM algebras $A_{1} \oplus A_{1}, A_{2}$ and $H_{2}(q, q)(q>2)$ were given.

We note that the problem of integrability of Toda chain equations corresponding to (nonsingular) KM algebras arises also in the context of fluxbrane solutions [44] that have also a polynomial structure of moduli functions for finite-dimensional Lie algebras (see also [104]). (For similar $S$-brane solutions governed by polynomial functions and its applications in connection with cosmological problems see [105, 106, 107].)

Here we have considered only the case of non-degenerate matrix $A$. It is an open problem to find general classes of solutions with branes for the degenerate case when $\operatorname{det} A=0$ (e.g. corresponding to affine KM algebras). Some special solutions of such type with maximal set of composite electric $S$-branes (e.g. when $A$ is not obviously a generalized Cartan matrix) were 
found in $[108,109]$ and generalized in $[110,111]$ for arbitrary (anti-)self-dual parallel charge density form of dimension $2 m$ defined on Ricci-flat Riemannian sub-manifold of dimension $4 m$. In these examples the restrictions on brane intersections (2.11) and (2.12) were replaced by more general condition on the stress-energy tensor: $T_{N}^{M}=0, M \neq N$.

It should be noted here that the solutions related to Lorentzian (e.g. hyperbolic) KM algebras considered in Examples 4.1-5.1 are the new ones. (In Example 4.3 the brane configuration from $[96,6])$ was used.)

\section{Acknowledgements}

This work was supported in part by the Russian Foundation for Basic Research grant Nr. 07-02$13624-$ of $_{\text {tss }}$. We are grateful to D. Singleton for reading the manuscript and valuable comments. We are also indebted to anonymous referees whose comments have led to the improvement of the paper.

\section{References}

[1] Kac V.G., Simple irreducible graded Lie algebras of finite growth, Izv. Akad. Nauk SSSR Ser. Mat. 32 (1968), 1323-1367.

[2] Moody R.V., A new class of Lie algebras, J. Algebra 10 (1968), 211-230.

[3] Kac V.G., Infinite-dimensional Lie algebras, 3rd ed., Cambridge University Press, Cambridge, 1990.

[4] Fuchs J., Schweigert C., Symmetries, Lie algebras and representations. A graduate course for physicists, Cambridge Monographs on Mathematical Physics, Cambridge University Press, Cambridge, 1997.

[5] Nikulin V.V., On the classification of hyperbolic root systems of rank three, Proc. Steklov Inst. Math. 230 (2000), no. 3, 1-241, alg-geom/9711032, alg-geom/9712033, math.AG/9905150.

[6] Henneaux M., Persson D., Spindel P., Spacelike singularities and hidden symmetries of gravity, Living Rev. Relativity 11 (2008), lrr-2008-1, 232 pages, arXiv:0710.1818.

[7] Green M.B., Schwarz J.H., Witten E., Superstring theory, Cambridge University Press, Cambridge, 1987.

[8] Saclioglu C., Dynkin diagram for hyperbolic Kac-Moody algebras, J. Phys. A: Math. Gen. 22 (18) (1989), 3753-3769.

[9] de Buyl S., Schomblond C., Hyperbolic Kac-Moody algebras and Einstein biliards, J. Math. Phys. 45 (2004), 4464-4492, hep-th/0403285.

[10] Feingold A., Frenkel I.B., A hyperbolic Kac-Moody Algebra and the theory of Siegel modular forms of genus 2, Math. Ann. 263 (1983), 87-144.

[11] Julia B., Kac-Moody symmetry of gravitation and supergravity theories, in Applications of Group Theory in Physics and Mathematical Physics (Chicago, 1982), Lectures in Appl. Math., Vol. 21, Amer. Math. Soc., Providence, RI, 1985, 355-373.

[12] Mizoguchi S., $E_{10}$ symmetry in one-dimensional supergravity, Nuclear Phys. B 528 (1998), 238-264, hep-th/9703160.

[13] Nicolai H., A hyperbolic Lie algebra from supergravity, Phys. Lett. B 276 (1992), 333-340.

[14] Moore G., String duality, automorphic forms, and generalized Kac-Moody algebras, in String Theory, Gauge Theory and Quantum Gravity (Trieste, 1997). Nuclear Phys. Proc. Suppl. 67 (1998), 56-67, hep-th/9710198.

[15] Damour T., Henneaux M., $E_{10}, B E_{10}$ and arithmetical chaos in superstring cosmology, Phys. Rev. Lett. 86 (2001), 4749-4752, hep-th/0012172.

[16] Belinskii V.A., Lifshitz E.M., Khalatnikov I.M., Oscillating regime of approaching to peculiar point in relyativistic cosmology, Usp. Fiz. Nauk 102 (1970), 463-500 (in Russian).

Belinskii V.A., Lifshitz E.M., Khalatnikov I.M., A general solution of the Einstein equations with a time singularity, Adv. Phys. 31 (1982), 639-667.

[17] Damour T., Henneaux M., Chaos in superstring cosmology, Phys. Rev. Lett. 85 (2000), 920-923, hep-th/0003139.

[18] Damour T., Henneaux M., Julia B., Nicolai H., Hyperbolic Kac-Moody algebras and chaos in Kaluza-Klein models, Phys. Lett. B 509 (2001), 323-330, hep-th/0103094. 
[19] Chitre D.M., Investigations of vanishing of a horizon for Bianchy type IX (the Mixmaster) universe, Ph.D. Thesis, University of Maryland, 1972.

[20] Ivashchuk V.D., Kirillov A.A., Melnikov V.N., On stochastic properties of multidimensional cosmological models near the singular point, Izv. Vyssh. Uchebn. Zaved. Fiz. 37 (1994), no. 11, 107-111 (English transl.: Russian Phys. J. 37 (1994), 1102-1106).

[21] Ivashchuk V.D., Kirillov A.A., Melnikov V.N., On stochastic behaviour of multidimensional cosmological models near the singularity, Pis'ma ZhETF 60 (1994), no. 4, 225-229 (English transl.: JETP Lett. 60 (1994), 235-239).

[22] Ivashchuk V.D., Melnikov V.N., Billiard representation for multidimensional cosmology with multicomponent perfect fluid near the singularity, Classical Quantum Gravity 12 (1995), 809-826, gr-qc/9407028.

[23] Ivashchuk V.D., Melnikov V.N., Billiard representation for pseudo-Euclidean Toda-like systems of cosmological origin, Regul. Chaotic Dyn. 1 (1996), no. 2, 23-35.

[24] Ivashchuk V.D., Melnikov V.N., Billiard representation for multidimensional cosmology with intersecting p-branes near the singularity, J. Math. Phys. 41 (2000), 6341-6363, hep-th/9904077.

[25] Damour T., Henneaux M., Nicolai H., Cosmological billiards, Classical Quantum Gravity 20 (2003), R145R200, hep-th/0212256.

[26] Ivashchuk V.D., Melnikov V.N., On billiard approach in multidimensional cosmological models, Gravit. Cosmol. 15 (2009), 49-58, arXiv:0811.2786.

[27] Cremmer E., Julia B., Scherk J., Supergravity theory in eleven-dimensions, Phys. Lett. B 76 (1978), 409-412.

[28] Hull C.M., Townsend P.K., Unity of superstring dualities, Nuclear Phys. B 438 (1995), 109-137, hep-th/9410167.

[29] Witten E., String theory dynamics in various dimensions, Nuclear Phys. B 443 (1995), 85-126, hep-th/9503124.

[30] Damour T., Henneaux M., Nicolai H., $E_{10}$ and a small tension expansion of M-theory, Phys. Rev. Lett. 89 (2002), 221601, 4 pages, hep-th/0207267.

[31] Gaberdiel M.R., Olive D.I., West P.C., A class of Lorentzian Kac-Moody algebras, Nuclear Phys. B 645 (2002), 403-437, hep-th/0205068.

[32] West P., E $E_{11}$ and M theory, Classical Quantum Gravity 18 (2001), 4443-4460, hep-th/0104081.

[33] Schnakenburg I., West P., Kac-Moody symmetries of IIB supergravity, Phys. Lett. B 517 (2001), 421-428, hep-th/0107181.

[34] Lambert N.D., West P.C., Coset symmetries in dimensionally reduced bosonic string theory, Nuclear Phys. B 615 (2001), 117-132, hep-th/0107209.

[35] Englert F., Houart L., Taormina A., West P., The symmetry of M-theories, J. High Energy Phys. 0309 (2003), no. 9, 020, 39 pages, hep-th/0304206.

[36] Englert F., Houart L., West P., Intersection rules, dynamics and symmetries, J. High Energy Phys. 2003 (2003), no. 8, 025, 26 pages, hep-th/0307024.

[37] Ivashchuk V.D., Melnikov V.N., Majumdar-Papapetrou-type solutions in sigma-model and intersecting $p$ branes, Classical Quantum Gravity 16 (1999), 849-869, hep-th/9802121.

[38] Grebeniuk M.A., Ivashchuk V.D., Sigma-model solutions and intersecting $p$-branes related to Lie algebras, Phys. Lett. B 442 (1998), 125-135, hep-th/9805113.

[39] Ivashchuk V.D., Kim S.-W., Melnikov V.N., Hyperbolic Kac-Moody algebra from intersecting $p$-branes, J. Math. Phys. 40 (1998), 4072-4083, Erratum, J. Math. Phys. 42 (2001), 5493, hep-th/9803006.

[40] Ivashchuk V.D., Kim S.-W., Solutions with intersecting p-branes related to Toda chains, J. Math. Phys. 41 (2000), 444-460, hep-th/9907019.

[41] Ivashchuk V.D., Melnikov V.N., p-brane black holes for general intersections, Gravit. Cosmol. 5 (1999), 313-318, gr-qc/0002085.

[42] Ivashchuk V.D., Melnikov V.N., Black hole p-brane solutions for general intersection rules, Gravit. Cosmol. 6 (2000), 27-40, hep-th/9910041.

[43] Ivashchuk V.D., Melnikov V.N., Toda $p$-brane black holes and polynomials related to Lie algebras, Classical Quantum Gravity 17 (2000), 2073-2092, math-ph/0002048.

[44] Ivashchuk V.D., Composite fluxbranes with general intersections, Classical Quantum Gravity 19 (2002), 3033-3047, hep-th/0202022. 
[45] Ivashchuk V.D., Composite S-brane solutions related to Toda-type systems, Classical Quantum Gravity 20 (2003), 261-275, hep-th/0208101.

[46] Ivashchuk V.D., Melnikov V.N., Exact solutions in multidimensional gravity with antisymmetric forms, Classical Quantum Gravity 18 (2001), R82-R157, hep-th/0110274.

[47] Ivashchuk V.D., Melnikov V.N., Multidimensional classical and quantum cosmology with intersecting $p$ branes, J. Math. Phys., 39 (1998), 2866-2888, hep-th/9708157.

[48] Ivashchuk V.D., Melnikov V.N., Sigma-model for the generalized composite p-branes, Classical Quantum Gravity 14 (1997), 3001-3029, Corrigenda, Classical Quantum Gravity 15 (1998), 3941-3942, hep-th/9705036.

[49] Englert F., Houart L., $G^{+++}$invariant formulation of gravity and M-theories: exact intersecting brane solutions, J. High Energy Phys. 2004 (2004), no. 5, 059, 15 pages, hep-th/0405082.

[50] Ivashchuk V.D., Melnikov V.N., Intersecting $p$-brane solutions in multidimensional gravity and M-theory, Gravit. Cosmol. 2 (1996), 297-305, hep-th/9612089.

[51] Ivashchuk V.D., Melnikov V.N., Generalized intersecting p-brane solutions from $\sigma$-model, Phys. Lett. B 403 (1997), 23-30.

[52] Ivashchuk V.D., Melnikov V.N., Rainer M., Multidimensional sigma-models with composite electric p-branes, Gravit. Cosmol. 4 (1998), 73-82, gr-qc/9705005.

[53] Aref'eva I.Ya., Rytchkov O.A., Incidence matrix description of intersecting p-brane solutions, in L.D. Faddeev's Seminar on Mathematical Physics, Amer. Math. Soc. Transl. Ser. 2, Vol. 201, Providence, RI, 2000, 19-38, hep-th/9612236.

[54] Argurio R., Englert F., Hourant L., Intersection rules for p-branes, Phys. Lett. B 398 (1997), 61-68, hep-th/9701042.

[55] Aref'eva I.Ya., Ivanov M.G., Rytchkov O.A., Properties of intersecting $p$-branes in various dimensions, in Supersymmetry and Quantum Field Theory (Kharkov, 1997), Lecture Notes in Phys., Vol. 509, Springer, Berlin, 1998, 25-41 hep-th/9702077.

[56] Aref'eva I.Ya., Ivanov M.G., Volovich I.V., Non-extremal intersecting $p$-branes in various dimensions, Phys. Lett. B 406 (1997), 44-48, hep-th/9702079.

[57] Ohta N., Intersection rules for non-extreme p-branes, Phys. Lett. B 403 (1997), 218-224, hep-th/9702164.

[58] Bronnikov K.A., Ivashchuk V.D., Melnikov V.N., The Reissner-Nordström problem for intersecting electric and magnetic p-branes, Gravit. Cosmol. 3 (1997), 203-212, gr-qc/9710054.

[59] Ivashchuk V.D., On symmetries of target space for $\sigma$-model of p-brane origin, Gravit. Cosmol. 4 (1998), 217-220, hep-th/9804102.

[60] Gal'tsov D.V., Rytchkov O.A., Generating branes via sigma models, Phys. Rev. D 58 (1998), 122001, 13 pages, hep-th/9801180.

[61] West P., The IIA, IIB and eleven dimensional theories and their common $E_{11}$ origin, Nuclear Phys. B 693 (2004), 76-102, hep-th/0402140.

[62] Tseytlin A.A., Harmonic superposition of M-branes, Nuclear Phys. B 475 (1996), 149-163, hep-th/9604035.

[63] Bergshoeff E., de Roo M., Eyras E., Janssen B., van der Schaar J.P., Multiple intersections of D-branes and M-branes, Nuclear Phys. B 494 (1997), 119-143, hep-th/9612095.

[64] Gauntlett J.P., Intersecting branes, in Proceeding of the February 97 APCTP Winter School on Dualities of Gauge and String Theories, World Sci. Publ., River Edge, NJ, 1998, 146-193, hep-th/9705011.

[65] Tseytlin A.A., No-force condition and BPS combinations of p-branes in 11 and 10 dimensions, Nuclear Phys. B 487 (1997), 141-154, hep-th/9609212.

[66] Gibbons G.W., Hawking S.W., Action integrals and partition functions in quantum gravity, Phys. Rev. D 15 (1977), 2752-2751.

[67] Ivashchuk V.D., Melnikov V.N., Zhuk A.I., On Wheeler-DeWitt equation in multidimensional cosmology, Nuovo Cimento B 104 (1989), 575-582.

[68] Nicolai H., Olive D.I., The principal SO(1,2) subalgebra of a hyperbolic Kac-Moody algebra, Lett. Math. Phys. 58 (2001), 141-152, hep-th/0107146.

[69] Khviengia N. , Khviengia Z., Lü H., Pope C.N., Towards a field theory of F-theory, Classical Quantum Gravity 15 (1998), 759-773, hep-th/9703012.

[70] Vafa C., Evidence for F-theory, Nuclear Phys. B 469 (1996), 403-415, hep-th/9602022. 
[71] Majumdar S.D., A class of exact solutions of Einstein's field equations, Phys. Rev. 72 (1947), 390-398. Papapetrou A., A static solution of the gravitational field for arbitrary charge distribution, Proc. Roy. Irish Acad. Sect. A 51 (1947), 191-204.

[72] Gavrilov V.R., Melnikov V.N., Toda chains with type $A_{m}$ Lie algebra for multidimensional classical cosmology with intersecting $p$-branes, in Proceedings of the International seminar "Curent Topics in Mathematical Cosmology" (30 March - 4 April, 1998, Potsdam, Germany), Editors M. Rainer and H.-J. Schmidt, World Scientific, 1998, 310-322, hep-th/9807004.

[73] Gavrilov V.R., Melnikov V.N., D-dimensional p-brane cosmological models associated with Lie algebras of type $A_{m}$, Theoret. and Math. Phys. 123 (2000), 726-743.

[74] Lü H., Mukherji S., Pope C.N., Xu K.-W., Cosmological solutions in string theories, Phys. Rev. D 55 (1997), 7926-7935, hep-th/9610107.

[75] Lü H., Pope C.N., Xu K.W., Liouville and Toda solitons in M-theory, Modern Phys. Lett. A 11 (1996), 1785-1796, hep-th/9604058.

[76] Lü H., Maharana H., Mukherji S., Pope C.N., Cosmological solutions, p-branes and the Wheeler-DeWitt equation, Phys. Rev. D 57 (1997), 2219-2229, hep-th/9707182.

[77] Gutperle M., Strominger A., Spacelike branes, J. High Energy Phys. 2002 (2002), no. 4, 018,19 pages, hep-th/0202210.

[78] Chen C.M., Gal'tsov D.M., Gutperle M., S-brane solutions in supergravity theories, Phys. Rev. D 66 (2002), 024043, 6 pages, hep-th/0204071.

[79] Kruczenski M., Myers R.C., Peet A.W., Supergravity S-branes, J. High Energy Phys. 2002 (2002), no. 5, 039, 23 pages, hep-th/0204144.

[80] Roy S., On supergravity solutions of space-like Dp-branes, J. High Energy Phys. 2002 (2002), no. 8, 025, 13 pages, hep-th/0205198.

[81] Degger N.S., Kaya A., Intersecting S-brane solutions of D = 11 supergravity, J. High Energy Phys. 2002 (2002), no. 7, 038, 14 pages, hep-th/0206057.

[82] Ohta N., Intersection rules for S-branes, Phys. Lett. B 558 (2003), 213-220, hep-th/0301095.

[83] Ivashchuk V.D., On composite S-brane solutions with orthogonal intersection rules, hep-th/0309027.

[84] Bronnikov K.A., Block-orthogonal brane systems, black holes and wormholes, Gravit. Cosmol. 4 (1998), 49-56, hep-th/9710207.

[85] Ivashchuk V.D., Melnikov V.N., Cosmological and spherically symmetric solutions with intersecting $p$-branes, J. Math. Phys. 40 (1999), 6558-6576.

[86] Ivashchuk V.D., Melnikov V.N., Multidimensional cosmological and spherically symmetric solutions with intersecting $p$-branes, in Mathematical and Quantum Aspects of Relativity and Cosmology, Proceedings of the Second Samos Meeting on Cosmology, Geometry and Relativity (1998, Pythagoreon, Samos, Greece), Editors S. Cotsakis and G.W. Gibbons, Lecture Notes in Phys., Vol. 537, Springer, Berlin, 2000, 214-247, gr-qc/9901001.

[87] Toda M., Waves in nonlinear lattice, Progr. Theor. Phys. Suppl. 45 (1970), 174-200.

[88] Bogoyavlensky O.I., On perturbations of the periodic Toda lattice, Comm. Math. Phys. 51 (1976), 201-209.

[89] Kostant B., The solution to a generalized Toda lattice and representation theory, Adv. in Math. 34 (1979), 195-338.

[90] Olshanetsky M.A., Perelomov A.M., Explicit solutions of the periodic Toda lattices, Invent. Math. 54 (1979), 261-269.

[91] Adler M., van Moerbeke P., Kowalewski's asymptotic method, Kac-Moody Lie algebras and regularization, Comm. Math. Phys. 83 (1982), 83-106.

[92] Kozlov V.V., Treshchev D.V., Polynomial integrals of Hamiltonian systems with exponential interaction, Math. USSR-Izv. 34 (1990), 555-574.

[93] Emel'yanov K.V., Tsygvintsev A.V., Kovalevskaya exponents of systems with exponential interaction, $S b$. Math. 191 (2000), 1459-1469.

[94] Gebert R.W., Inami T., Mizoguchi S., Toda field theories associated with hyperbolic Kac-Moody algebra Painlevé properties and $W$ algebras, Internat. J. Modern Phys. A 11 (1996), 5479-5493, hep-th/9503176.

[95] Fre P., Sorin A.S., The arrow of time and the Weyl group: all supergravity billiards are integrable, arXiv:0710.1059. 
[96] Henneaux M., Leston M., Persson D., Spindel P., Geometric configurations, regular subalgebras of $E_{10}$ and M-theory cosmology, J. High Energy Phys. 2006 (2006), no. 10, 021, 49 pages, hep-th/0606123.

[97] Ivashchuk V.D., Melnikov V.N., Multidimensional classical and quantum cosmology with perfect fluid, Gravit. Cosmol. 1 (1995), 133-148, hep-th/9503223.

[98] Alimi J.-M., Ivashchuk V.D., Kononogov S.A., Melnikov V.N., Multidimensional cosmology with anisotropic fluid: acceleration and variation of G, Gravit. Cosmol. 12 (2006), 173-178, gr-qc/0611015.

[99] Cvetic M., Tseytlin A.A., Nonextreme black holes from nonextreme intersecting M-branes, Nuclear Phys. B 478 (1996), 181-198, hep-th/9606033.

[100] Ohta N., Shimizu T., Non-extreme black holes from intersecting M-branes, Internat. J. Modern Phys. A 13 (1998), 1305-1328, hep-th/9701095.

[101] Cotsakis S., Ivashchuk V.D., Melnikov V.N., p-brane black holes and post-Newtonian approximation, Gravit. Cosmol. 5 (1999), 52-57, gr-qc/9804080.

[102] Grebeniuk M.A., Ivashchuk V.D., Kim S.-W., Black-brane solutions for $C_{2}$ algebra, J. Math. Phys. 43 (2002), 6016-6023, hep-th/0111219.

[103] Grebeniuk M.A., Ivashchuk V.D., Melnikov V.N., Black-brane solution for $A_{3}$ algebra, Phys. Lett. B 543 (2002), 98-106, hep-th/0208083.

[104] Golubtsova A.A., Ivashchuk V.D., On calculation of fluxbrane polynomials corresponding to classical series of Lie algebras, arXiv:0804.0757.

[105] Goncharenko I.S., Ivashchuk V.D., Melnikov V.N., Fluxbrane and S-brane solutions with polynomials related to rank-2 Lie algebras, Gravit. Cosmol. 13 (2007), 262-266, math-ph/0612079.

[106] Alimi J.-M., Ivashchuk V.D., Melnikov V.N., An S-brane solution with acceleration and small enough variation of $G$, Gravit. Cosmol. 13 (2007), 137-141, arXiv:0711.3770.

[107] Ivashchuk V.D., Kononogov S.A., Melnikov V.N., Electric S-brane solutions corresponding to rank-2 Lie algebras: acceleration and small variation of G, Grav. Cosmol. 14, No. 3 (2008), 235-240, arXiv:0901.0025.

[108] Ivashchuk V.D., Singleton D., Composite electric S-brane solutions with maximal number of branes, J. High Energy Phys. 2004 (2004), no. 10, 061, 18 pages, hep-th/0407224.

[109] Ivashchuk V.D., Melnikov V.N., Singleton D., On avoiding cosmological oscillating behavior for S-brane solutions with diagonal metrics, Phys. Rev. D 72 (2005), 103511, 8 pages, gr-qc/0509065.

[110] Ivashchuk V.D., Melnikov V.N., Singleton D., Electric S-brane solutions with parallel forms on Ricci-flat factor space, Gravit. Cosmol. 12 (2006), 315-320, hep-th/0610167.

[111] Dehnen H., Ivashchuk V.D., Melnikov V.N., S-brane solutions with (anti-)self-dual parallel charge density form on a Ricci-flat submanifold, Gravit. Cosmol. 13 (2007), 23-30, hep-th/0612260. 